\title{
Common BRCA1 and BRCA2 Mutations among Latin American Breast Cancer Subjects: A Meta-Analysis
}

Leonardo M Porchia'1, M Elba González Mejia², Luis Calderilla-Barbosa', Nirvana I Ordaz Diaz', Fabiola Islas Lugo', José Oldak¹, Rossana C Zepeda ${ }^{3}$ and Gisela Aguirre ${ }^{1 *}$

${ }^{1}$ Laboratorio de Genética y Biología Molecular, D-SU Biotek S.A. de C.V, México

${ }^{2}$ Facultad de Medicina, Benemérita Universidad Autónoma de Puebla, México

${ }^{3}$ Centro de Investigaciones Biomedicas, Universidad Veracruzana, México

\begin{abstract}
Background: Many BRCA1 and BRCA2 mutations have been characterized in breast cancer subjects; however the overall prevalence in Latin American remains elusive. The aim of the study was to determine the prevalence of common BRCA1 and BRCA2 mutations in Latin American breast cancer subjects.

Methods: Pubmed, EBSCO, and OVID databases, and study bibliographies were systematically searched for observational studies that examined for mutations in BRCA1 and BRCA2 until March 2015. The pooled prevalence was obtained using the inverse double arcsine square root method. Publication bias was assessed by Begg and Mazumdar's test and the Egger's test. The sensitivity was determined by reevaluation of the pooled estimate after removal of one study.

Results: Out of 294 retrieved studies, 32 studies met the inclusion criteria ( $n=9938$ subjects). Twenty-nine BRCA1 and thirteen BRCA2 pathogenic mutations were described in two or more studies. For BRCA1, the most reported mutations were 185delAG and A1708E. The most prevalent BRCA1 mutations $(>0.50 \%)$ were del exon 9-12 (1.45\%, 95\% Cl: 0.61-2.63\%), 185delAG (0.90\%, 95\% Cl: 0.50-1.42\%), R71G (0.64\%, 95\% Cl: 0.43-0.87\%), A1708E (0.58\%, 95\% Cl: 0.40-0.79\%), 5382insC (0.54\%, 95\% Cl: 0.32-0.82\%), and del exon $16-17$ (0.54\%, 95\% Cl: $0.32-0.82 \%)$. For BRCA2, the most reported mutations were 6174 delT and $3036 \mathrm{del} 4$; however, the $\mathrm{H} 372 \mathrm{~N}(0.78 \%$, $95 \% \mathrm{Cl}: 0.14-1.82 \%)$ was the most frequent $(>0.50 \%)$. Comparing Mexican-based studies to the remaining Latin American reports, we provide evidence that certain mutations are specific only for Mexicans and their descendants (i.e. BRCA1 del exon 9-12 and BRCA2 3492insT, G273R, and W2586X).
\end{abstract}

Conclusion: Here we identify the most common BRCA1 and BRCA2 mutations among Latin Americans. This information will aid in selecting mutations for genetic testing and in epidemiological studies.

Keywords: BRCA1; BRCA2; Latin America; Meta-analysis; Breast cancer; Polymorphism; Mexico

\section{Introduction}

Breast cancer is the most common cancer among Latin American women and the leading cause of cancer-associated deaths $[1,2]$. It is estimated that 114,900 new cases and 37,000 deaths occur in Latin American populations annually [3]. Unfortunately, Latin American women have a poor 5 year survival rate than most other ethnic groups [4] and the incidence is increasing annually in these countries [57]. Genetic cancer risk assessment has become an integral part of disease prevention, especially in countries such as Spain and USA; however, the limited availability of clinical gene testing has prevented the implementation of prevention programs in Central and South American countries.

Five to ten percent of all breast cancers in Latin American women are attributed to germ-line mutations in the breast cancer susceptibility genes BRCA1 and BRCA2 [8,9]. Conversely, in low-income countries with restricted financial resources for genetic testing, this percentage has been suggested to be underestimated. The lifetime risk of developing breast cancer increases up to $80 \%$ with certain BRCA1 and BRCA2 mutations [10]. BRCA mutations prevalence varies between country as well as ethnic groups [11]. With more than 300 documented BRCA1 and BRCA2 mutations found in Hispanic countries [12-22] and with limited reports describing the prevalence of BRCA1 and BRCA2 mutations, which can range from $0 \%$ to over $50 \%$, we therefore conducted a meta-analysis to determine the prevalence of certain pathogenic mutations in Central and South American countries.

\section{Methods and Materials}

\section{Publication search}

Pubmed, OVID, and EBSCO databases were searched for all studies that investigated the prevalence of BRCA1 and BRCA2 mutations found among Latin American breast cancer individuals. The following keywords and index terms were used: "Latin, Hispanic, South and Central America" as well as other terms associated with all Latin American countries, "BRCA1 and BRCA2", and "deletion, insertion, mutation, and polymorphism" for any publication published up to March 30, 2015. Only papers published in English, Spanish, and Portuguese were reviewed. Afterwards, the complied publications' references were hand searched. The titles and abstract were reviewed and reports that were not eligible for this study were eliminated. All

*Corresponding author: Gisela Aguirre, Laboratorio de Genética y Biología Molecular, D-SU Biotek S.A. de C.V. Paseo de los Heroes 10231 Int.301 Zona Urbana Rio, 22010, Tijuana, Baja California, México, Tel: 01 (55) 1227 2200; E-mail: gisi@dsubiotek.com

Received June 02, 2015; Accepted June 22, 2015; Published June 24, 2015

Citation: Porchia LM, Gonzalez-Mejia ME, Calderilla-Barbosa L, Ordaz-Diaz $\mathrm{N}$, Islas $\mathrm{F}$, et al. (2015) Common BRCA1 and BRCA2 Mutations among Latin American Breast Cancer Subjects: A Meta-Analysis. J Carcinogene Mutagene 6: 228. doi:10.4172/2157-2518.1000228

Copyright: (C) 2015 Porchia LM, et al. This is an open-access article distributed under the terms of the Creative Commons Attribution License, which permits unrestricted use, distribution, and reproduction in any medium, provided the original author and source are credited. 
studies had to meet the following criteria: studies focused on examining the prevalence of BRCA1 or BRCA2 mutations in human subjects, with breast cancer, from Latin American countries or their descendants. Non-human studies, in vitro or in vivo studies, reviews, studies that failed to indicate the prevalence of mutations, or focused on other than breast cancer were excluded.

\section{Data extraction}

Two of the authors extracted all data independently. If there was a disagreement, another author assessed the publication in question. If a single sample was believed to be use in multiple publications, the publications were assessed to determine which one was the most representative and that data was used for that mutation, or the corresponding author was contacted to resolve the issue. The data collected were geographical location, criteria use to select sample, sample size, the mutation and the number of positive individuals, method used to detect the mutations, and exons and introns examined.

\section{Statistical analysis}

The term "Prevalence" corresponds to relative prevalence rate, which was defined as the total number of positive individuals with breast cancer for a specific mutation divided by the total number of breast cancer cases. The prevalence and the $95 \%$ confidence interval (95\% CI) were calculated for each mutation. Next, the pooled prevalence estimate was calculated using the inverse double arcsine square root method (Stuart-Ord) [23]. It is worthy to note that only studies that examined the exon or the intron of the BRCA1 or BRCA2 gene or specifically indicated they examined for the mutation of interest were included in the meta-analysis, independent if the authors found the mutation or not. Heterogeneity was determined using the $\psi^{2}$-based Q test and its degree was assessed by the $\mathrm{I}^{2}$ value (inconsistency index). The Fixed Effects Model was used when the sample was considered homogeneous (Mantel-Haenszel method) [24] and the random effects model was used when the sample was considered heterogeneous (DerSimonian and Laird Method) [25]. The stability and sensitivity of the results were assessed by removing one study and re-calculating the pooled prevalence. Publication bias was evaluated by Begg and Mazumdar adjusted rank correlation asymmetry test (Kendall's tau) and the Egger regression asymmetry test $[26,27]$. The Fisher's exact test was used to determined difference of frequencies between groups. Statistical analyses were performed using StatDirect Statistical Software version 3.0.147 (Cheshire, UK). P-values $<0.05$ (two-sided) were considered statistically significant.

\section{Results}

\section{Characteristic of the studies}

Using the search terms, we identified 294 possible studies from the multiple databases and from reviewing study's bibliographies. We excluded 241 studies that focused on cancers other than Breast cancer $(n=22)$, did not focus on Latin Americans $(n=6)$, did not focus on human subjects $(n=24)$, the BRCA genes were not the focus of the study $(n=92)$, did not determine the frequency of BRCA mutations $(n=74)$ or were not a research article $(n=23)$. The remaining 53 studies were extensively evaluated. Thirteen studies were excluded because they lacked sufficient information and eight more studies were excluded due to shared samples. This led to 32 studies, consisting of 9938 subjects, which were included for the meta-analysis (Figure 1). Detailed characteristics of these studies are presented in Table 1. The most represented country

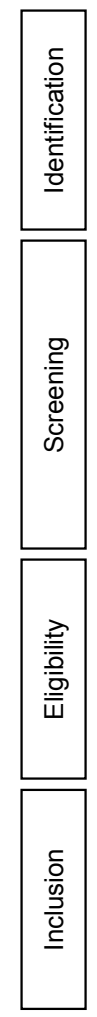

294 studies retrieved by search terms "Latin, Hispanic, South and Central America" as well as other terms associated with all Latin American countries, "BRCA1 and BRCA2", and "deletion, insertion, mutation, and polymorphism" from Pubmed, EBSCO, and OVID databases and study bibliographies.

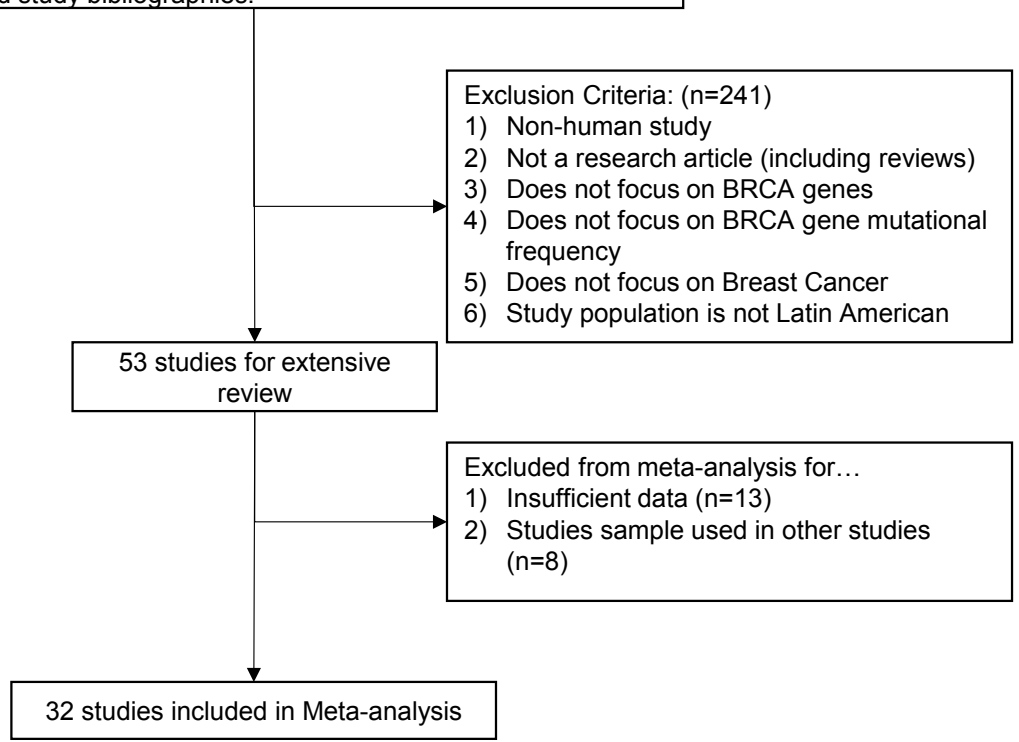

Figure 1: Meta-analysis. 
Citation: Porchia LM, Gonzalez-Mejia ME, Calderilla-Barbosa L, Ordaz-Diaz N, Islas F, et al. (2015) Common BRCA1 and BRCA2 Mutations among Latin American Breast Cancer Subjects: A Meta-Analysis. J Carcinogene Mutagene 6: 228. doi:10.4172/2157-2518.1000228

Page 3 of 12

\begin{tabular}{|c|c|c|c|c|c|c|c|}
\hline \multirow{2}{*}{$\begin{array}{l}\text { Author, Year } \\
\text { Country }\end{array}$} & \multirow{2}{*}{ BRCA Coverage } & \multirow{2}{*}{ Screening Method } & \multicolumn{2}{|c|}{ \# of mutations ${ }^{a}$} & \multirow{2}{*}{$\mathrm{N}$} & \multirow{2}{*}{ Study inclusion criteria } & \multirow{2}{*}{ Ref. } \\
\hline & & & BRCA1 & BRCA2 & & & \\
\hline $\begin{array}{l}\text { Abugattas, } 2014 \\
\text { Peru }\end{array}$ & HISPANEL-114 mutations & Sequencing & 4 & 1 & 266 & $\begin{array}{l}\text { unselected breast cancer patients, diagnosed at } \\
\text { any age }\end{array}$ & [49] \\
\hline $\begin{array}{l}\text { Anton Culver, } 2000 \\
\text { USA-Hispanics }\end{array}$ & $\begin{array}{l}\text { BRCA1: 185delAG, 5382insC, } \\
\text { R1443X, int5-11T-G, R841W, } \\
\text { 4184delTCAA, 2594delC }\end{array}$ & ASO & 0 & $\mathrm{~N} / \mathrm{A}$ & 42 & $\begin{array}{l}\text { unselected breast cancer patients, greater than } 18 \\
\text { years old }\end{array}$ & {$[51]$} \\
\hline $\begin{array}{l}\text { Carraro, } 2011 \\
\text { Brazil }\end{array}$ & $\begin{array}{l}\text { BRCA1 and BRCA2: All exons and } \\
\text { adjacent intronic regions }\end{array}$ & Sequencing & 8 & 10 & 54 & $\begin{array}{l}\text { unselected breast cancer patients, aged } 35 \text { years } \\
\text { or younger at the time of diagnosis }\end{array}$ & [33] \\
\hline $\begin{array}{l}\text { Delgado, } 2011 \\
\text { Uruguay }\end{array}$ & $\begin{array}{l}\text { BRCA1 and BRCA2: All exons and } \\
\text { adjacent intronic regions }\end{array}$ & $\begin{array}{l}\text { HDA, PTT, } \\
\text { Sequencing }\end{array}$ & 7 & 14 & 42 & $\begin{array}{l}\text { 1) at least three cases of female breast cancer } \\
\text { with at least one of them diagnosed before the age } \\
\text { of } 50 \\
\text { 2) two cases of female breast cancer at least } \\
\text { one diagnosed before } 50 \text { years old and at least } \\
\text { one of the following additional criteria: paternal } \\
\text { transmission, bilateral breast cancer, ovarian } \\
\text { cancer, male breast cancer. }\end{array}$ & {$[50]$} \\
\hline $\begin{array}{l}\text { Dufloth, } 2005 \\
\text { Brazil }\end{array}$ & $\begin{array}{l}\text { BRCA1: exon 2, 3, 5, 11, } 20 \\
\text { BRCA2: exon 10, 11" }\end{array}$ & SSCP, sequencing & 1 & 2 & 31 & $\begin{array}{l}\text { 1) early onset (at less than } 45 \text { years of age) and/ } \\
\text { or bilaterality } \\
\text { 2) more than three cases of breast cancer and } \\
\text { more than one case of ovarian cancer in the family } \\
\text { 3) more than two first-degree relatives involved } \\
\text { 4) male breast cancer }\end{array}$ & [34] \\
\hline $\begin{array}{l}\text { Esteves, } 2009 \\
\text { Brazil }\end{array}$ & $\begin{array}{l}\text { BRCA1: exon } 11 \\
\text { BRCA2: exon } 10,11\end{array}$ & PTT, sequencing & 7 & 3 & 612 & $\begin{array}{l}\text { 1) women with a history of two or more relatives } \\
\text { with breast and/or ovarian cancer along three or } \\
\text { more generations } \\
\text { 2) two or more cases of breast and/or ovarian } \\
\text { cancer in first-degree relatives } \\
\text { 3) male relatives with breast cancer diagnosed } \\
\text { under } 35 \text { years of age } \\
\text { 4) cases of bilateral breast cancer }\end{array}$ & [35] \\
\hline $\begin{array}{l}\text { Ewald, } 2011 \\
\text { Brazil }\end{array}$ & $\begin{array}{l}\text { BRCA1: 185delAG, 5382insC } \\
\text { BRCA2: 6174delT }\end{array}$ & PCR & 1 & 0 & 131 & $\begin{array}{l}\text { 1) the American Society of Clinical Oncology } \\
\text { (ASCO) criteria for HBOC } \\
\text { 2) a prior probability of harboring a BRCA mutation } \\
\geq 30 \% \text { by pedigree analysis using the Myriad } \\
\text { mutation prevalence tables or the Penn II mutation } \\
\text { prediction model } \\
\text { 3) Women diagnosed with bilateral breast cancer } \\
\text { under the age of } 50 \text { years, regardless of family } \\
\text { history }\end{array}$ & {$[36]$} \\
\hline $\begin{array}{l}\text { Gallardo, } 2006 \\
\text { Chile }\end{array}$ & $\begin{array}{l}\text { BRCA1 and BRCA2: covering } \\
\text { coding sequences and intron-exon } \\
\text { boundaries (exons } 2 \text { to } 24 \text { ) }\end{array}$ & $\begin{array}{l}\text { PCR, SSCP, PTT, } \\
\text { HDA, sequencing }\end{array}$ & 15 & 11 & 54 & $\begin{array}{l}\text { 1) three cases of breast cancer in first degree } \\
\text { relatives } \\
\text { 2) two cases of breast cancer in first degree } \\
\text { relatives, one diagnosed before age } 40 \\
\text { 3) one breast cancer and one ovarian cancer in } \\
\text { first degree relatives }\end{array}$ & [39] \\
\hline $\begin{array}{l}\text { Garcia-Jimenez, } 2012 \\
\text { Costa Rica }\end{array}$ & $\begin{array}{l}\text { BRCA1: exon } 11 \\
\text { BRCA2: exon } 10,11\end{array}$ & PTT & 1 & 2 & 116 & $\begin{array}{l}\text { 1) least three individuals diagnosed with breast } \\
\text { cancer at any age or ovarian cancer on one side } \\
\text { of the family } \\
\text { 2) at least two individuals diagnosed with breast } \\
\text { cancer under the age of } 50 \text { on one side of the } \\
\text { family }\end{array}$ & {$[48]$} \\
\hline $\begin{array}{l}\text { Gomes, } 2007 \\
\text { Brazil }\end{array}$ & $\begin{array}{l}\text { BRCA1: exon } 11 \\
\text { BRCA2: exon } 10,11\end{array}$ & $\mathrm{PTT}$, sequencing & 2 & 2 & 402 & unselected breast cancer patients & {$[37]$} \\
\hline $\begin{array}{l}\text { Gonzalez- } \\
\text { Hormazabal, } 2011 \\
\text { Chile }\end{array}$ & $\begin{array}{l}\text { BRCA1 and BRCA2: All exons and } \\
\text { adjacent intronic regions }\end{array}$ & $\begin{array}{l}\text { CSGE, HDA, } \\
\text { Sequencing }\end{array}$ & 30 & 25 & 326 & $\begin{array}{l}\text { 1) Three or more family members with breast and/ } \\
\text { or ovarian cancer } \\
\text { 2) Two family members with breast cancer } \\
\text { 3) One family member with breast cancer and one } \\
\text { with ovarian cancer } \\
\text { 4) Single affected individual with breast cancer } \\
\text { greater than or equal to age } 35 \\
\text { 5) Single affected individual with breast cancer } \\
\text { greater than or equal to age } 50 \\
\text { 6) Single affected individual with male breast } \\
\text { cancer }\end{array}$ & {$[40]$} \\
\hline $\begin{array}{l}\text { Hall, } 2009 \\
\text { USA-Hispanics }\end{array}$ & $\begin{array}{l}\text { BRCA1 and BRCA2: All exons and } \\
\text { adjacent intronic regions }\end{array}$ & Myraid ${ }^{b}$ & 5 & 2 & 1936 & $\begin{array}{l}\text { Persons undergoing clinical full-sequence for } \\
\text { BRCA1/2 mutations at Myraid Genetics Inc. from } \\
\text { November } 1996 \text { to March } 2006\end{array}$ & {$[16]$} \\
\hline $\begin{array}{l}\text { Hernandez, } 2014 \\
\text { Colombia }\end{array}$ & $\begin{array}{l}\text { BRCA1: exon } 11 \\
\text { BRCA2: exon } 10,11\end{array}$ & PTT, sequencing & 2 & 1 & 244 & $\begin{array}{l}\text { unselected breast cancer patients, diagnosed at } \\
\text { any age }\end{array}$ & [43] \\
\hline
\end{tabular}


Citation: Porchia LM, Gonzalez-Mejia ME, Calderilla-Barbosa L, Ordaz-Diaz N, Islas F, et al. (2015) Common BRCA1 and BRCA2 Mutations among Latin American Breast Cancer Subjects: A Meta-Analysis. J Carcinogene Mutagene 6: 228. doi:10.4172/2157-2518.1000228

\begin{tabular}{|c|c|c|c|c|c|c|c|}
\hline $\begin{array}{l}\text { John, } 2007 \\
\text { USA-Hispanics }\end{array}$ & $\begin{array}{l}\text { BRCA1: All exons and adjacent } \\
\text { intronic regions }\end{array}$ & $\begin{array}{l}\text { CSGE, 2DGS, } \\
\text { Myraid b }\end{array}$ & 11 & $\mathrm{~N} / \mathrm{A}$ & 393 & $\begin{array}{l}\text { A: patients whose cancers are likely to be } \\
\text { hereditary } \\
\text { 1) breast cancer diagnosis before age } 35 \text { years } \\
\text { 2) bilateral breast cancer, with first diagnosis } \\
\text { before age } 50 \text { years } \\
\text { 3) prior ovarian or childhood cancer } \\
\text { 4) at least } 1 \text { first-degree relative with breast or } \\
\text { ovarian cancer. } \\
\text { B: all other patients with cancers less likely to be } \\
\text { hereditary }\end{array}$ & [52] \\
\hline $\begin{array}{l}\text { Judkins, } 2012 \\
\text { USA-Hispanics }\end{array}$ & $\begin{array}{l}\text { BRCA1 and BRCA2: All Large } \\
\text { genomic rearrangements }\end{array}$ & $\begin{array}{l}\text { multiplex PCR, } \\
\text { Myraid }^{\mathrm{b}}\end{array}$ & & & & $\begin{array}{l}\text { 1) invasive or in situ breast cancer diagnosed } \\
\text { under age } 50 \text { years, or ovarian cancer } \\
\text { 2)male breast cancer diagnosed at any age, in } \\
\text { conjunction with } 2 \text { or more relatives similarly } \\
\text { affected } \\
\text { 3) breast cancer patient with no deleterious } \\
\text { mutation detected by BRCA } 1 / 2 \text { sequencing }\end{array}$ & [53] \\
\hline $\begin{array}{l}\text { Lara, } 2012 \\
\text { Venezuela }\end{array}$ & $\begin{array}{l}\text { BRCA1: All exons and adjacent } \\
\text { intronic regions }\end{array}$ & CSGE, sequencing & 24 & 30 & 58 & $\begin{array}{l}\text { 1) early onset (less than } 45 \text { years of age) and/or } \\
\text { bilaterality } \\
\text { 2) more than three cases of breast cancer and } \\
\text { more than one case of ovarian cancer in the family } \\
\text { 3) more than two first-degree relatives affected } \\
\text { 4) male breast cancer }\end{array}$ & [17] \\
\hline $\begin{array}{l}\text { Nahleh, } 2015 \\
\text { Mexico }\end{array}$ & $\begin{array}{l}\text { BRCA1 and BRCA2: All exons and } \\
\text { adjacent intronic regions }\end{array}$ & Myraid $^{b}$ & 11 & 5 & 88 & $\begin{array}{l}\text { unselected breast cancer patients, diagnosed at } \\
\text { any age }\end{array}$ & [28] \\
\hline $\begin{array}{l}\text { Ruiz-Flores, } 2002 \\
\text { Mexico }\end{array}$ & $\begin{array}{l}\text { BRCA1 and BRCA2: All exons and } \\
\text { adjacent intronic regions }\end{array}$ & HDA, Sequencing & 3 & 7 & 51 & $\begin{array}{l}\text { 1) breast cancer diagnosed at age } 35 \text { or younger, } \\
\text { with no first or second-degree relatives affected } \\
\text { with breast or ovarian cancer } \\
\text { 2) two cases or more of breast cancer with at least } \\
\text { one case diagnosed under } 60 \text { among first and } \\
\text { second-degree relatives } \\
\text { 3) one case of breast cancer under } 60 \text { and one } \\
\text { case of ovarian cancer diagnosed at any age in } \\
\text { first and second degree relatives } \\
\text { 4) one case of female and one case of male } \\
\text { breast cancer diagnosed at any age in first degree } \\
\text { relatives }\end{array}$ & [18] \\
\hline $\begin{array}{l}\text { Sanabria, } 2009 \\
\text { Colombia }\end{array}$ & BRCA1: 185delAG, 5382insC & PCR & 0 & $\mathrm{~N} / \mathrm{A}$ & 30 & $\begin{array}{l}\text { 1) Woman of any age with diagnostics of BC } \\
\text { 2) With Family background in first or second } \\
\text { degree } \\
\text { 3) Woman with diagnostics of BC under } 36 \text { year's } \\
\text { old, Without Family background }\end{array}$ & [44] \\
\hline $\begin{array}{l}\text { Sanchez, } 2011 \\
\text { Chile }\end{array}$ & $\begin{array}{l}\text { BRCA1 and BRCA2: only Large } \\
\text { Genomic rearrangements }\end{array}$ & MLPA & 2 & 0 & 74 & $\begin{array}{l}\text { 1) three cases of breast cancer in first degree } \\
\text { relatives } \\
\text { 2) two cases of breast cancer in first degree } \\
\text { relatives, one diagnosed before age } 40 \\
\text { 3) one breast cancer and one ovarian cancer in } \\
\text { first degree relatives }\end{array}$ & [41] \\
\hline $\begin{array}{l}\text { Silva, } 2014 \\
\text { Brazil }\end{array}$ & $\begin{array}{l}\text { BRCA1 and BRCA2: All exons and } \\
\text { adjacent intronic regions }\end{array}$ & Sequencing, MLPA & 18 & 6 & 120 & $\begin{array}{l}\text { 1) Breast cancer diagnosed } \leq 45 \text { years of age (no } \\
\text { family history) } \\
\text { 2) Breast cancer diagnosed } \leq 45 \text { years of age with } \\
1 \text { or more close blood relative with breast/ovarian/ } \\
\text { fallopian tube/primary peritoneal cancer at any age } \\
\text { 3) Breast cancer diagnosed }<45 \leq 50 \text { years of age } \\
\text { with } 1 \text { or more blood relative with breast/ovarian/ } \\
\text { fallopian tube/primary peritoneal cancer } \leq 50 \text { years } \\
\text { of age } \\
\text { 4) Breast cancer diagnosed }>50 \text { of age with } 1 \text { or } \\
\text { more blood relative with breast/ovarian/fallopian } \\
\text { tube/primary peritoneal cancer at any age } \\
\text { 5) Two primary BC when the first occurrence was } \\
\text { prior to age } 50 \\
\text { 6) Breast cancer with a history of ovarian/ fallopian } \\
\text { tube/primary peritoneal cancer at any age } \\
\text { 7) For an individual with an ethnicity that is } \\
\text { associated with a higher mutation frequency (e.g., } \\
\text { Ashkenazi Jewish) } \\
\text { 8) Personal history of ovarian/fallopian tube/ } \\
\text { primary peritoneal cancer } \\
\text { 9) Personal history of male breast cancer }\end{array}$ & [38] \\
\hline $\begin{array}{l}\text { Solano, } 2012 \\
\text { Argentina }\end{array}$ & $\begin{array}{l}\text { BRCA1 and BRCA2: All exons and } \\
\text { adjacent intronic regions }\end{array}$ & Sequencing & 36 & 23 & 134 & $\begin{array}{l}\text { 1) diagnosis within } 40 \text { years of age and no BOC } \\
\text { family history } \\
\text { 2) diagnosis at any age with at least two BOC } \\
\text { affected } 1 \text { st or } 2 \text { nd degree relatives }\end{array}$ & [47] \\
\hline
\end{tabular}




\begin{tabular}{|c|c|c|c|c|c|c|c|}
\hline $\begin{array}{l}\text { Torres, } 2007 \\
\text { Colombia }\end{array}$ & $\begin{array}{l}\text { BRCA1 and BRCA2: All exons and } \\
\text { adjacent intronic regions }\end{array}$ & $\begin{array}{l}\text { SSCP, PTT, DHPLC, } \\
\text { sequencing }\end{array}$ & 4 & 9 & 44 & $\begin{array}{l}\text { 1) one female breast cancer diagnosed at or } \\
\text { before } 35 \text { years of age. } \\
\text { 2) two cases of breast cancer diagnosed at any } \\
\text { age. } \\
\text { 3) three cases of breast cancer with at least one } \\
\text { diagnosed at or before } 50 \text { years of age. } \\
\text { 4) at least four breast cancers with as a minimum } \\
\text { one diagnosed at or before } 50 \text { years of age. } \\
\text { 5) at least one male breast cancer diagnosed at } \\
\text { any age. } \\
6 \text { ) at least one female breast cancer and one or } \\
\text { more ovarian cancers at any age }\end{array}$ & {$[45]$} \\
\hline $\begin{array}{l}\text { Torres, } 2009 \\
\text { Colombia }\end{array}$ & BRCA1: del exon 9-12 & PCR & 0 & $\mathrm{~N} / \mathrm{A}$ & 229 & $\begin{array}{l}\text { unselected breast cancer patients, diagnosed at } \\
\text { any age }\end{array}$ & {$[46]$} \\
\hline $\begin{array}{l}\text { Torres-Mejia, } 2014 \\
\text { Mexico }\end{array}$ & $\begin{array}{l}\text { "BRCA1: exon 10, 5382insC, del } \\
\text { exon9-12, 185delAG R1443X, } \\
\text { A1708E, C1787S \& G1788D, } \\
\text { BRCA2: exon 10, } 11 \text { W2586X }\end{array}$ & $\begin{array}{l}\text { ARMS, CNV, } \\
\text { MLPA, PTT, RFLP, } \\
\text { sequencing }\end{array}$ & 8 & 11 & 810 & $\begin{array}{l}\text { unselected breast cancer patients, diagnosed at } \\
\text { any age }\end{array}$ & [29] \\
\hline $\begin{array}{l}\text { Vaca-Paniagua, } 2012 \\
\text { Mexico }\end{array}$ & $\begin{array}{l}\text { BRCA1 and BRCA2: All exons and } \\
\text { adjacent intronic regions }\end{array}$ & Pyrosequencing & 13 & 10 & 39 & $\begin{array}{l}\text { 1) subjects with breast and/or ovarian cancer and } \\
\text { with two or more first- or second-degree relatives } \\
\text { with tumors associated with BRCA mutations were } \\
\text { studied. } \\
\text { 2) male breast cancer. } \\
\text { 3) Subjects with early-onset breast cancer } \\
\text { 4) subjects with breast and ovarian cancer }\end{array}$ & [19] \\
\hline $\begin{array}{l}\text { Vidal-Millan, } 2009 \\
\text { Mexico }\end{array}$ & $\begin{array}{l}\text { BRCA1 and BRCA2: All exons and } \\
\text { adjacent intronic regions }\end{array}$ & $\begin{array}{l}\text { PCR, DHPLC, } \\
\text { sequencing }\end{array}$ & 8 & 6 & 40 & $\begin{array}{l}\text { 1) BC diagnosis at age } 40 \text { or younger, with no first } \\
\text { or second-degree relatives } \\
\text { 2) at least } 2 \text { cases of } B C \text { and/or OV at any age in } \\
\text { first-degree relatives. } \\
\text { 3) one case of male BC diagnosed at any age in } \\
\text { first-degree relatives. }\end{array}$ & {$[30]$} \\
\hline $\begin{array}{l}\text { Villarreal-Garza, } \\
2015 a \\
\text { Mexico }\end{array}$ & HISPANEL = 115 mutations & Sequencing & 10 & 1 & 190 & $\begin{array}{l}\text { Patients diagnosed with triple-negative breast } \\
\text { cancer at age } 50 \text { years or younger. }\end{array}$ & [31] \\
\hline $\begin{array}{l}\text { Villarreal-Garza, } \\
2015 b \\
\text { Mexico }\end{array}$ & HISPANEL = 114 mutations & $\begin{array}{l}\text { Pyrosequencing, } \\
\text { sequencing, MPLA }\end{array}$ & 8 & 3 & 96 & $\begin{array}{l}\text { unselected breast cancer patients, diagnosed at } \\
\text { any age }\end{array}$ & [32] \\
\hline $\begin{array}{l}\text { Vogel, } 2007 \\
\text { USA-Hispanics }\end{array}$ & $\begin{array}{l}\text { BRCA1 and BRCA2: All exons and } \\
\text { adjacent intronic regions }\end{array}$ & Myraid $^{b}$ & 11 & 9 & 95 & Subjects had a personal or family history of $B C$ & {$[20]$} \\
\hline $\begin{array}{l}\text { Weitzel, } 2013 \\
\text { USA-Hispanics }\end{array}$ & $\begin{array}{l}\text { BRCA1 and BRCA2: All exons and } \\
\text { adjacent intronic regions }\end{array}$ & Myraid $^{b}$ & 11 & 4 & 746 & $\begin{array}{l}\text { Subjects with a personal or family history of BC } \\
\text { and/or OV }\end{array}$ & [21] \\
\hline
\end{tabular}

aNumber of different mutations identified

'Mutational analysis was done at Myraid Genetic, Inc.

Abbreviations: ARMS: Amplification Refractory Mutation System; ASO: Allele-Specific Oligonucleotide Assays; BC: Breast Cancer; CNV: Copy Number Variation Assay; CSGE: Conformation-Specific Gel Electrophoresis; 2DGS: 2-Dimensional Gene Scanning; DHPLC: Denaturing High-Pressure Liquid Chromatography; HDA: Heteroduplex Analyses; MLPA: Multiplex Ligation-Dependent Probe Amplification; OV: Ovarian Cancer; PTT: Protein Truncation Test; RFLP: Restriction Fragment Length Polymorphism; SSCP: Single Strand Conformational Polymorphism

Table 1: Characteristics of included studies.

\begin{tabular}{|l|c|c|c|c|}
\hline \multirow{2}{*}{ Type of Mutation } & \multicolumn{2}{|c|}{ BRCA1 } & \multicolumn{2}{c|}{ BRCA2 } \\
\cline { 2 - 5 } & ALL & Pathogenic & ALL & Pathogenic \\
\hline Latin Americans & & & & \\
\hline Deletion & 51 & 36 & 41 & 34 \\
\hline Insertion & 18 & 11 & 16 & 10 \\
\hline Single Nucleotide variant & 105 & 33 & 103 & 24 \\
\hline Mixed & 1 & 0 & 2 & 1 \\
\hline Total & 175 & 80 & 162 & 69 \\
\hline & & & & \\
\hline Mexicans & & & & \\
\hline Deletion & 19 & 18 & 15 & 7 \\
\hline Insertion & 3 & 3 & 4 & 1 \\
\hline Single Nucleotide variant & 35 & 12 & 33 & 4 \\
\hline Mixed & 0 & 0 & 1 & 0 \\
\hline Total & 57 & 33 & 53 & 12 \\
\hline
\end{tabular}

in this study was Mexico ( $\mathrm{n}=7 ;[18,19,28-32])$, followed by Brazil $(\mathrm{n}=6$; [33-38]). Chile [39-42] and Colombia [43-46] both had four studies included in this meta-analysis and Argentina [47], Costa Rica [48], Peru [49], Uruguay [50], and Venezuela [17] each had one study. Six studies focused on Latin American subjects from multiple countries and their descendants $[16,20,21,51,52]$. The 32 studies used fourteen different methods to screen from BRCAs mutations: sequencing $(n=18)$, protein truncation test (PTT, $n=8)$, PCR $(n=6)$, heteroduplex analyses (HDA, $\mathrm{n}=4$ ), multiplex ligation-dependent probe amplification (MPLA, $n=4$ ), conformation-specific gel electrophoresis (CSGE, $n=3$ ), denaturing high-pressure liquid chromatography (DHPLC, $n=3$ ), single strand conformational polymorphism (SSCP, $\mathrm{n}=3$ ), pyrosequencing $(\mathrm{n}=2)$, 2-dimensional gene scanning (2DGS, $\mathrm{n}=1$ ), amplification refractory mutation system (ARMS, $n=1$ ), allele-specific oligonucleotide assays $(\mathrm{ASO}, \mathrm{n}=1)$, copy number variation assay $(\mathrm{CNV}, \mathrm{n}=1)$, and restriction fragment length polymorphism (RFLP, $\mathrm{n}=1$ ). Six studies used Myriad Genetics, Inc. to screen for BRCA mutations and large genomic

Table 2: Distribution of BRCA1 and BRCA2 mutation among Latin Americans and Mexicans. 
Citation: Porchia LM, Gonzalez-Mejia ME, Calderilla-Barbosa L, Ordaz-Diaz N, Islas F, et al. (2015) Common BRCA1 and BRCA2 Mutations among Latin American Breast Cancer Subjects: A Meta-Analysis. J Carcinogene Mutagene 6: 228. doi:10.4172/2157-2518.1000228

Page 6 of 12

\begin{tabular}{|c|c|c|c|c|c|c|c|c|c|c|c|c|c|c|c|c|}
\hline \multirow[b]{3}{*}{ Mutation } & \multicolumn{16}{|c|}{ Total Latin Americans } \\
\hline & \multirow[b]{2}{*}{$\begin{array}{c}\text { \# of } \\
\text { studies }\end{array}$} & \multirow[b]{2}{*}{$\mathrm{n} / \mathrm{N}$} & \multirow[b]{2}{*}{ Freq $(95 \% \mathrm{Cl}), \%$} & \multicolumn{3}{|c|}{ Heterogeneity } & \multicolumn{2}{|c|}{ Publication Bias } & \multirow[b]{2}{*}{$\begin{array}{c}\text { \# of } \\
\text { studies }\end{array}$} & \multirow[b]{2}{*}{$\mathrm{n} / \mathrm{N}$} & \multirow[b]{2}{*}{ Freq $(95 \% \mathrm{Cl}), \%$} & \multicolumn{3}{|c|}{ Heterogeneity } & \multicolumn{2}{|c|}{ Publication Bias } \\
\hline & & & & Q-test & $I^{2}$-test & Model & $\begin{array}{c}\text { Begg's } \\
\text { test }\end{array}$ & $\begin{array}{c}\text { Egger's } \\
\text { test }\end{array}$ & & & & Q-test & $1^{2}$-test & Model & $\begin{array}{c}\text { Begg's } \\
\text { test }\end{array}$ & $\begin{array}{c}\text { Egger's } \\
\text { test }\end{array}$ \\
\hline \multicolumn{17}{|l|}{ BRCA1 } \\
\hline All pathogenic & 32 & $215 / 9938$ & $5.70(4.12-7.53)$ & $<0.01$ & $90.80 \%$ & RE & 0.04 & 0.01 & 9 & 185/2096 & $8.60(3.68-15.31)$ & $<0.01$ & $94.70 \%$ & RE & 0.48 & 0.08 \\
\hline del exon 9-12 & 11 & $8 / 4811$ & $1.45(0.61-2.63)$ & $<0.01$ & $83.3 \%$ & $\mathrm{RE}$ & 0.22 & 0.50 & 5 & $44 / 1676$ & $3.35(1.18-6.57)$ & $<0.01$ & $87.0 \%$ & RE & 0.48 & 0.13 \\
\hline 185delAG & 29 & 17245 & $0.90(0.50-1.42)$ & $<0.01$ & $68.5 \%$ & RE & 0.04 & 0.05 & 9 & $22 / 1906$ & $0.94(0.18-2.29)$ & $<0.01$ & $79.0 \%$ & RE & 0.76 & 0.14 \\
\hline R71G & 21 & $30 / 5007$ & $0.64(0.43-0.87)$ & 0.53 & $0.0 \%$ & $\mathrm{FE}$ & 0.09 & 0.71 & 7 & $12 / 1086$ & $1.24(0.67-1.98)$ & 0.73 & $0.0 \%$ & $\mathrm{FE}$ & 0.56 & 0.36 \\
\hline A1708E & 21 & $32 / 5786$ & $0.58(0.40-0.79$ & 0.27 & $14.2 \%$ & $\mathrm{FE}$ & 0.37 & 0.53 & 8 & 10/1896 & $0.67(0.35-1.08)$ & 0.98 & $0.0 \%$ & $\mathrm{FE}$ & 0.72 & 1.00 \\
\hline 5382insC & 28 & $25 / 6554$ & $0.54(0.25-0.94)$ & $<0.01$ & $61.4 \%$ & RE & $<0.01$ & 0.01 & 8 & $1 / 1380$ & $0.13(0.01-0.39)$ & 0.61 & $0.0 \%$ & $\mathrm{FE}$ & $<0.01$ & 0.33 \\
\hline del exon 16-17 & 7 & $18 / 3369$ & $0.54(0.32-0.82)$ & 0.19 & $31.8 \%$ & FE & 0.56 & 0.92 & 3 & $1 / 663$ & $0.17(0.00-0.62)$ & 0.19 & $40.7 \%$ & FE & N/A & N/A \\
\hline del exon $1-2$ & 7 & $21 / 3369$ & $0.46(0.10-1.09)$ & 0.06 & $51.0 \%$ & RE & 0.14 & 0.97 & 3 & $2 / 729$ & $0.51(0.01-2.37)$ & 0.04 & $68.1 \%$ & $\mathrm{RE}$ & N/A & N/A \\
\hline 2552delC & 25 & $20 / 6974$ & $0.34(0.22-0.49)$ & 0.88 & $0.0 \%$ & FE & 0.01 & 0.81 & 8 & 7/1896 & $0.46(0.21-0.81)$ & 0.82 & $0.0 \%$ & $\mathrm{FE}$ & 0.40 & 0.96 \\
\hline R1443X & 22 & $13 / 5828$ & $0.29(0.13-0.53)$ & 0.09 & $30.5 \%$ & RE & $<0.01$ & 0.10 & 8 & 11/1896 & $0.64(0.33-1.04)$ & 0.40 & $3.8 \%$ & $\mathrm{FE}$ & 0.72 & 0.46 \\
\hline 917delTT & 23 & $12 / 5735$ & $0.20(0.10-0.33)$ & 0.11 & $28.1 \%$ & FE & 0.01 & 0.06 & 7 & $6 / 1086$ & $0.68(0.28-1.25)$ & 0.80 & $0.0 \%$ & $\mathrm{FE}$ & 0.77 & 0.60 \\
\hline 3148delCT & 25 & $12 / 6947$ & $0.20(0.11-0.33)$ & 0.79 & $0.0 \%$ & $\mathrm{FE}$ & $<0.01$ & 0.83 & 8 & 1/1896 & $0.10(0.01-0.29)$ & 0.61 & $0.0 \%$ & $\mathrm{FE}$ & 0.01 & 0.31 \\
\hline 943ins 10 & 25 & $8 / 6311$ & $0.12(0.05-0.22)$ & 0.17 & $21.4 \%$ & FE & $<0.01$ & 0.07 & 8 & $6 / 1380$ & $0.65(0.01-1.76)$ & 0.02 & $59.4 \%$ & $\mathrm{RE}$ & 0.06 & 0.23 \\
\hline 3450del4 & 25 & $11 / 6974$ & $0.15(0.07-0.25)$ & 0.11 & $26.3 \%$ & FE & $<0.01$ & 0.15 & 8 & 0/1896 & N/A & & & & & \\
\hline Q1200X & 25 & $8 / 6947$ & $0.15(0.07-0.25)$ & 0.76 & $0.0 \%$ & $\mathrm{FE}$ & $<0.01$ & 0.18 & 8 & 7/1896 & $0.45(0.20-0.80)$ & 0.71 & $0.0 \%$ & FE & 0.55 & 0.71 \\
\hline C61G & 21 & $4 / 5007$ & $0.12(0.04-0.23)$ & 0.67 & $0.0 \%$ & FE & $<0.01$ & 0.05 & 7 & 0/1086 & $\mathrm{N} / \mathrm{A}$ & & & & & \\
\hline 3878delTA & 25 & $6 / 6947$ & $0.11(0.05-0.20)$ & 0.51 & $0.0 \%$ & $\mathrm{FE}$ & $<0.01$ & 0.02 & 8 & $4 / 1896$ & $0.39(0.06-0.99)$ & 0.08 & $44.9 \%$ & RE & 0.55 & 0.08 \\
\hline 2925del4 & 25 & $7 / 6311$ & $0.11(0.04-0.20)$ & 0.21 & $17.8 \%$ & FE & $<0.01$ & 0.04 & 8 & $7 / 1380$ & $0.93(0.17-2.29)$ & 0.01 & $63.1 \%$ & RE & 0.18 & 0.06 \\
\hline C1787S/D1788G & 22 & $6 / 6398$ & $0.11(0.04-0.20)$ & 0.52 & $0.0 \%$ & $\mathrm{FE}$ & $<0.01$ & 0.21 & 8 & $6 / 1896$ & $0.40(0.07-0.97)$ & 0.09 & $41.8 \%$ & RE & 0.18 & 0.28 \\
\hline s955X & 25 & $5 / 6947$ & $0.11(0.03-0.17)$ & 0.83 & $0.0 \%$ & FE & $<0.01$ & 0.27 & 8 & 4/1896 & $0.23(0.07-0.50)$ & 0.41 & $3.0 \%$ & $\mathrm{FE}$ & 0.06 & 0.56 \\
\hline 1129insA & 25 & 4/6311 & $0.10(0.04-0.20)$ & 0.82 & $0.0 \%$ & FE & $<0.01$ & 0.13 & 8 & $2 / 1380$ & $0.26(0.06-0.60)$ & 0.86 & $0.0 \%$ & $\mathrm{FE}$ & 0.18 & 0.83 \\
\hline 2415delAG & 25 & $3 / 6311$ & $0.10(0.03-0.20)$ & 0.96 & $0.0 \%$ & FE & $<0.01$ & 0.15 & 8 & $3 / 1380$ & $0.34(0.10-0.71)$ & 0.80 & $0.0 \%$ & FE & 0.11 & 0.47 \\
\hline K654X & 25 & 2/6311 & $0.09(0.03-0.18)$ & 0.98 & $0.0 \%$ & $\mathrm{FE}$ & $<0.01$ & 0.19 & 8 & $1 / 1380$ & $0.13(0.01-0.39)$ & 0.61 & $0.0 \%$ & FE & $<0.01$ & 0.33 \\
\hline S1040N & 23 & $4 / 6083$ & $0.08(0.03-0.17)$ & 0.51 & $0.0 \%$ & $\mathrm{FE}$ & $<0.01$ & 0.06 & 7 & 1/1086 & $0.16(0.01-0.48)$ & 0.57 & $0.0 \%$ & $\mathrm{FE}$ & $<0.01$ & 0.30 \\
\hline 3977del4 & 25 & 2/6947 & $0.08(0.03-0.16)$ & 0.97 & $0.0 \%$ & FE & $<0.01$ & 0.17 & 8 & 1/1896 & $0.10(0.01-0.29)$ & 0.62 & $0.0 \%$ & FE & 0.01 & 0.31 \\
\hline 4184del4 & 26 & 2/6989 & $0.08(0.03-0.16)$ & 0.97 & $0.0 \%$ & FE & $<0.01$ & 0.17 & 8 & 1/1896 & $0.09(0.01-0.28)$ & 0.55 & $0.0 \%$ & $\mathrm{FE}$ & $<0.01$ & 0.27 \\
\hline Q1135X & 25 & 2/6947 & $0.08(0.03-0.16)$ & 0.98 & $0.0 \%$ & $\mathrm{FE}$ & $<0.01$ & 0.23 & 8 & 0/1896 & $N / A$ & & & & & \\
\hline R1751X & 22 & 2/5007 & $0.08(0.02-0.18)$ & 0.83 & $0.0 \%$ & $\mathrm{FE}$ & $<0.01$ & 0.13 & 7 & $1 / 1086$ & $17(0.01-0.50)$ & 0.63 & $0.0 \%$ & $\mathrm{FE}$ & 0.03 & 0.35 \\
\hline 2080delA & 25 & $3 / 6947$ & $0.08(0.02-0.16)$ & 0.89 & $0.0 \%$ & FE & $<0.01$ & 0.16 & 8 & 0/1896 & N/A & & & & & \\
\hline IVS7+2T>A & 13 & 2/3756 & $0.07(0.01-0.18)$ & 0.45 & $0.0 \%$ & $\mathrm{FE}$ & $<0.01$ & 0.15 & 3 & 837 & N/A & & & & & \\
\hline \multicolumn{17}{|l|}{ BRCA2 } \\
\hline All pathogenic & 28 & $215 / 9277$ & $31(2.54-6.52)$ & $<0.01$ & $94.7 \%$ & RE & 0.04 & $<0.01$ & 8 & $56 / 1896$ & $3.08(1.52-5.15)$ & $<0.01$ & $72.7 \%$ & $\mathrm{RE}$ & 0.40 & 0.32 \\
\hline $\mathrm{H} 372 \mathrm{~N}$ & 24 & $56 / 6554$ & $0.88(0.24-1.92)$ & $<0.01$ & $90.9 \%$ & RE & $<0.01$ & 0.11 & 8 & 0/1896 & N/A & & & & & \\
\hline E49X & 18 & $10 / 4339$ & $0.38(0.13-0.75)$ & 0.03 & $41.9 \%$ & RE & $<0.01$ & 0.07 & 7 & $6 / 1086$ & $0.68(0.28-1.25)$ & 0.77 & $0.0 \%$ & FE & 0.77 & 0.63 \\
\hline 3492ins $T$ & 24 & $24 / 6554$ & $0.37(0.24-0.53)$ & 0.29 & $12.1 \%$ & FE & $<0.01$ & 1.00 & 8 & $12 / 1896$ & $0.60(0.15-1.36)$ & 0.05 & $50.3 \%$ & $\mathrm{RE}$ & 0.18 & 0.52 \\
\hline 6174delT & 25 & $14 / 6725$ & $0.32(0.13-0.60)$ & $<0.01$ & $51.3 \%$ & RE & $<0.01$ & 0.03 & 8 & $0 / 1896$ & $\mathrm{~N} / \mathrm{A}$ & & & & & \\
\hline Q742X & 24 & $3 / 5918$ & $0.23(0.05-0.56)$ & 0.22 & $26.6 \%$ & FE & 0.28 & 0.09 & 8 & $3 / 1380$ & $0.09(0.03-0.18)$ & 0.83 & $0.0 \%$ & $\mathrm{FE}$ & $<0.01$ & 0.07 \\
\hline E1308X & 24 & $11 / 6554$ & $0.20(0.11-0.32)$ & 0.77 & $0.0 \%$ & $\mathrm{FE}$ & $<0.01$ & 0.92 & 8 & 0/1896 & N/A & & & & & \\
\hline G2793R & 18 & $5 / 4339$ & $0.14(0.05-0.27)$ & 0.54 & $0.0 \%$ & FE & $<0.01$ & 0.27 & 7 & $5 / 1086$ & $0.57(0.21-1.10)$ & 0.74 & $0.0 \%$ & $\mathrm{FE}$ & 0.24 & 0.76 \\
\hline 3036del4 & 24 & $7 / 6554$ & $0.11(0.05-0.21)$ & 0.29 & $12.6 \%$ & FE & $<0.01$ & 0.03 & 8 & $1 / 1896$ & $0.15(0.03-0.37)$ & 0.98 & $0.0 \%$ & $\mathrm{FE}$ & 0.06 & 0.67 \\
\hline W2586X & 18 & $2 / 3702$ & $0.10(0.02-0.23)$ & 0.76 & $0.0 \%$ & FE & $<0.01$ & 0.16 & 7 & $2 / 614$ & $0.56(0.11-1.33)$ & 0.70 & $0.0 \%$ & FE & 0.14 & 0.23 \\
\hline R3128X & 18 & $2 / 4339$ & $0.09(0.02-0.19)$ & 0.75 & $0.0 \%$ & $\mathrm{FE}$ & $<0.01$ & 0.15 & 7 & 0/1086 & $N / A$ & & & & & \\
\hline 6503delTT & 24 & $3 / 6554$ & $0.08(0.03-017)$ & 0.86 & $0.0 \%$ & $\mathrm{FE}$ & $<0.01$ & 0.16 & 8 & $0 / 1896$ & $\mathrm{~N} / \mathrm{A}$ & & & & & \\
\hline 3034del 4 & 24 & $2 / 6554$ & $0.07(0.02-0.15)$ & 0.92 & $0.0 \%$ & FE & $<0.01$ & 0.12 & 8 & $0 / 1896$ & $\mathrm{~N} / \mathrm{A}$ & & & & & \\
\hline 6714del4 & 24 & 2/6554 & $0.07(0.02-0.15)$ & 0.91 & $0.0 \%$ & $\mathrm{FE}$ & $<0.01$ & 0.12 & 8 & $1 / 1896$ & $0.10(0.01-0.29)$ & 0.62 & $0.0 \%$ & FE & 0.01 & 0.31 \\
\hline
\end{tabular}

Abbreviations: $n$ : Total Number of Subjects; N: Number of Subjects Positive for the Mutation; FE: Fixed-Effects Model; RE: Random-Effects Model; N/A: Not Applicable.

Table 3: Common BRCA1 and BRC2 pathogenic mutations identified in two or more studies.

rearrangements.

\section{Common BRCA1 mutations among Latin Americans}

Using the 32 studies, we identified 175 BRCA1 mutations found in Latin American breast cancer patients (Supplemental Table 1). A majority of mutations were single nucleotide variants $(n=105,60.0 \%$, Table 2). However, when only pathogenic mutations were identified $(\mathrm{n}=80,45.7 \%)$, by using the CLINVAR database or the studies themselves, 36 were deletions (45.0\%), 33 were single nucleotide variants $(41.25 \%)$, and 11 were insertions (13.75\%). Fifty pathogenic mutations $(62.5 \%)$ were reported only once, whereas fifteen mutations $(18.8 \%)$ were reported four times or more. The most identified mutation for BRCA1 were 185delAG and A1708E, found in eleven and ten studies, respectively.

The pathogenic mutations' pooled prevalence was calculated for mutations presenting two or more studies using the inverse double 
A

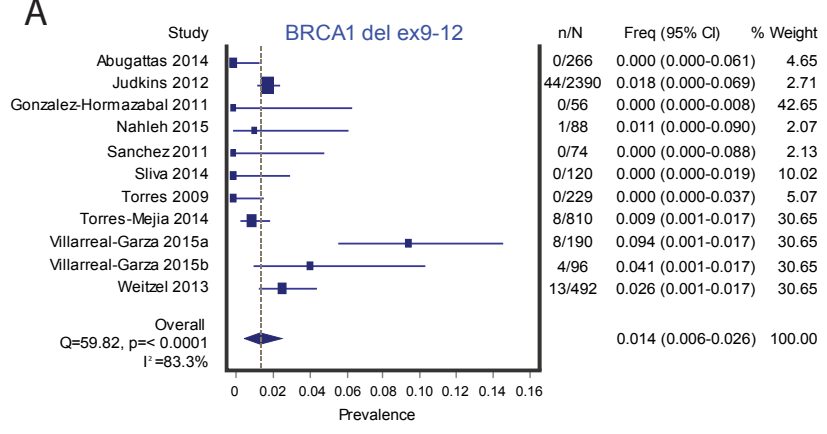

B

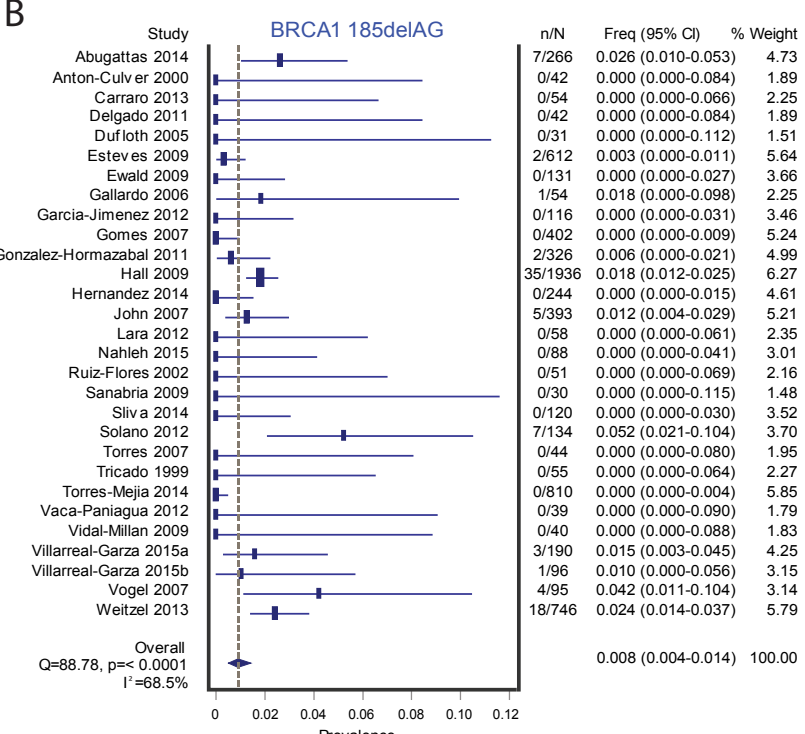

C

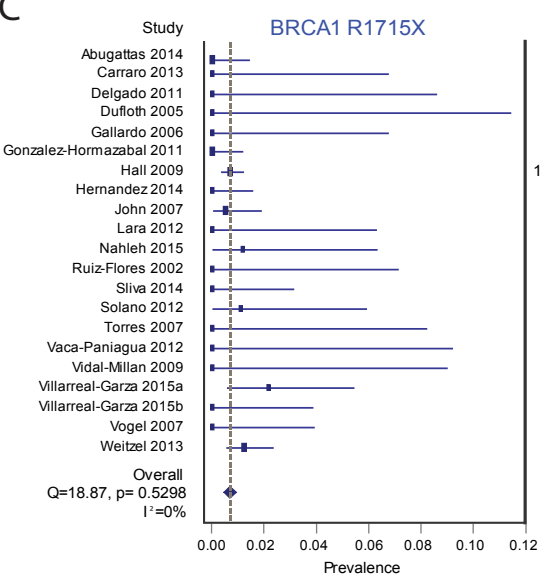

$\mathrm{n} / \mathrm{N} \quad$ Freq $(95 \% \mathrm{Cl}) \quad \%$ Weight $\begin{array}{lll}0 / 266 & 0.000(0.000-0.013) & 5.31 \\ 0.54 & 0.000(0.000-0.066) & 1.08\end{array}$ $0.42 \quad 0.000(0.000-0.084) \quad 0.84$ $\begin{array}{lll}0.000(0.000-0.112) & 0.62 \\ 0 / 54 & 0.000(0.000-0.066) & 1.08\end{array}$ $0 / 326 \quad 0.000(0.000-0.011) \quad 6.50$ $13 / 1936 \quad 0.006(0.003-0.011) \quad 38.59$ $\begin{array}{lll}0 / 244 & 0.000(0.000-0.015) & 4.87 \\ 2 / 393 & 0.005(0.000-0.018) & 7.84\end{array}$ $\begin{array}{ccc}2 / 393 & 0.005(0.000-0.018) & 7.84 \\ 0 / 58 & 0.000(0.000-0.061) & 1.16\end{array}$ $\begin{array}{lll} & & 0.58 \\ 1 / 88 & 0.011(0.000-0.061) & 1.16 \\ 0 & 0.061) & 1.76\end{array}$ $\begin{array}{lll}1 / 88 & 0.011(0.000-0.061) & 1.76 \\ 0 / 51 & 0.000(0.000-0.069) & 1.02\end{array}$ $\begin{array}{lll}0 / 120 & 0.000(0.000-0.030) & 2.40 \\ 104 & 0.010(0.000-057) & 1.88\end{array}$ $\begin{array}{lll}1 / 94 & 0.010(0.000-0.057) & 1.88 \\ 0 / 44 & 0.000(0.000-0.080) & 0.88 \\ 0 & 0.000(0.000-0.090) & 0.78\end{array}$ $\begin{array}{lll}0 / 39 & 0.000(0.000-0.090) & 0.78 \\ 0 / 40 & 0.000(0.000-0.088) & 0.80\end{array}$ $\begin{array}{ccc}0 / 40 & 0.000((0.000-0.088) & 0.88 \\ 4 / 190 & 0.021(0.005-0.053) & 3.79\end{array}$

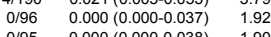
$9 / 746 \quad 0.012(0.005-0.022) \quad 14.87$ $0.006(0.004-0.008) \quad 100.00$

D

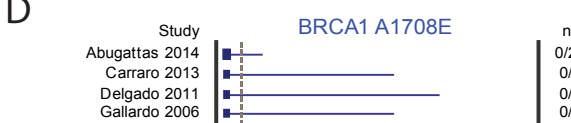

$\mathrm{n} / \mathrm{N} \quad$ Freq $(95 \% \mathrm{Cl}) \quad \%$ weight $\begin{array}{lll}0 / 266 & 0.000(0.000-0.013) & 4.59\end{array}$ $0.000(0.000-0.066) \quad 0.94$ $0 / 42 \quad 0.000(0.000-0.084) \quad 0.73$ $\begin{array}{rrr}0 / 54 & 0.000(0.000-0.066) & 0.94 \\ 0 / 326 & 0.000(0.000-0.011) & 5.63\end{array}$ $0 / 326 \quad 0.000(0.000-0.011) \quad 5.63$ $\begin{array}{ccr}14 / 1936 & 0.007(0.003-0.012) & 33.40 \\ 1 / 244 & 0.004(0.000-0.022) & 4.21 \\ 0 / 393 & 0.000(0.000-0.009) & 6.78\end{array}$

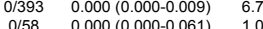

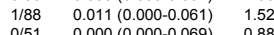
$\begin{array}{ccc}0 / 51 & 0.000(0.000-0.069) & 0.88 \\ 1 / 120 & 0.008(0.000-0.045) & 2.07\end{array}$ \begin{tabular}{ccc}
$1 / 120$ & $0.008(0.000-0.045)$ & 2.07 \\
$0 / 94$ & $0.000(0.000-0.038)$ & 1.63 \\
\hline & $0.068(0.014-0.186)$ & 0.76
\end{tabular} $\begin{array}{lll}0 / 94 & 0.000(0.000-0.038) & 1.63 \\ 3 / 44 & 0.068(0.014-0.186) & 0.76\end{array}$ $\begin{array}{ccr}3 / 44 & 0.068(0.014-0.186) & 0.76 \\ 4 / 810 & 0.004(0.001-0.012) & 13.98\end{array}$ $0 / 39 \quad 0.000(0.000-0.090) \quad 0.68$ $\begin{array}{lll}0 / 40 & 0.000(0.000-0.088) & 0.69\end{array}$

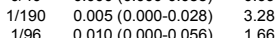
\begin{tabular}{lll}
$1 / 96$ & $0.010(0.000-0.056)$ & 1.66 \\
$2 / 95$ & $0.021(0.002-0.073)$ & 1.64 \\
\hline & 0.005
\end{tabular} $\begin{array}{rrr}2 / 95 & 0.021(0.002-0.073) & 1.64 \\ 4 / 746 & 0.005(0.001-0.013) & 12.87\end{array}$ Vidal-Millan 2009
Villarreal-Garza 2015 Villarreal-Garza 2015 Vogel 2007
Weitzel 2013 $\mathrm{Q}=23.29, \begin{array}{r}\text { Overall } \\ \mathrm{p}=0.2744 \\ \mathrm{I}^{2}=14.2 \%\end{array}$ $0.005(0.004-0.007) \quad 100.00$

Figure 2: BRCA1 mutations found in Latin American breast cancer subjects.

arcsine square root method. Meta-analysis results are presented in Table 3. The four most prevalent BRCA1 mutations found in Latin American breast cancer subjects were del exon 9-12 (1.45\%, 95\% CI: 0.61-2.63\%, Figure $2 \mathrm{~A}), 185$ delAG $(0.90 \%, 95 \%$ CI: $0.50-1.42 \%$, Figure $2 B)$, R71G (0.64\%, 95\% CI: $0.43-0.87 \%$, Figure $2 \mathrm{C})$, and A1708E (0.58\%, 95\% CI: $0.25-0.79 \%$, Figure 2D). The remaining Forest Plots can be found in supplemental material (Supplemental Figure 1).

Next, to determine if these mutations were consistent between groups, we examined the prevalence these mutations in a selected subgroup of subjects-Mexicans and Mexican descendants were selected because they had largest number of studies-and compared them to the rest of the Latin American population. Anton Culver 2000, Hall 2009, John 2007 (except for 185delAG analysis) and Vogel 2007 were excluded because Latin American origins could not be determined. The prevalence for the Mexican subjects for del exon 9-12 was 3.35\% (95\% CI: $1.18-6.57 \%$, Figure 3A), for 185delAG was $0.94 \%$ (95\% CI: $0.18-2.29 \%$, Figure $3 \mathrm{~B}$ ), for R71G was $1.24 \%$ (95\% CI: $0.67-1.98 \%$, Figure $3 \mathrm{C}$ ), and A1708E was $0.67 \%$ (95\% CI: 0.35-1.08\%, Figure 3D). The remaining Forest Plots can be found in supplemental material
(Supplemental Figure 2). Comparison of these mutations indicated that the frequency between Mexicans and other Latin American countries was not similar. The frequency of the R71G mutation was higher in Mexicans than other Latin Americans (Fisher's exact test, $\mathrm{p}=0.0034$ ), but there was no difference between the two groups for $185 \mathrm{delAG}$ $(\mathrm{p}=0.702)$ and $\mathrm{A} 1708 \mathrm{E}(\mathrm{p}=0.802)$. It is important to note that the deletion of BRCA1 exons 9-12 was only found in Mexican subjects.

\section{Common BRCA2 mutation among Latin Americans}

Twenty-five studies identified 162 mutations (Supplemental Table 2 ). The most common type of mutations were single nucleotide variants $(n=103,63.6 \%)$. Of the 162 mutations, only $69(42.6 \%)$ of these were pathogenic. Of the pathogenic mutations, the most common type were deletions $(n=34,49.3 \%)$ followed by single nucleotide variants $(n=24$, $34.8 \%)$. Thirteen mutations (18.8\%) were reported more than once. The most identified mutations were 6174delT and 3036del4, identified in six and five articles, respectively. The frequency of these mutations was determined by meta-analysis (Table 3). The most prevalent BRCA2 mutations found in Latin American Breast cancer subjects was H372N 


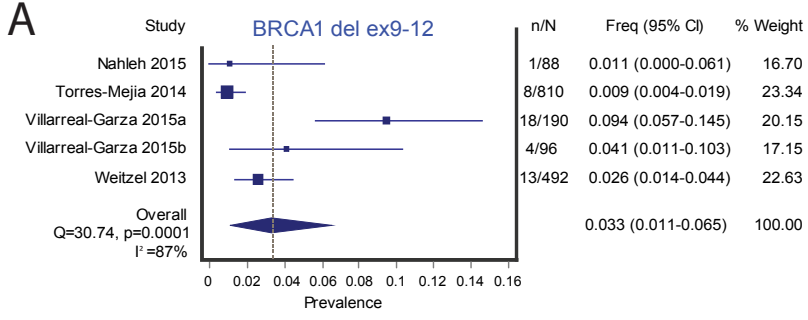

B

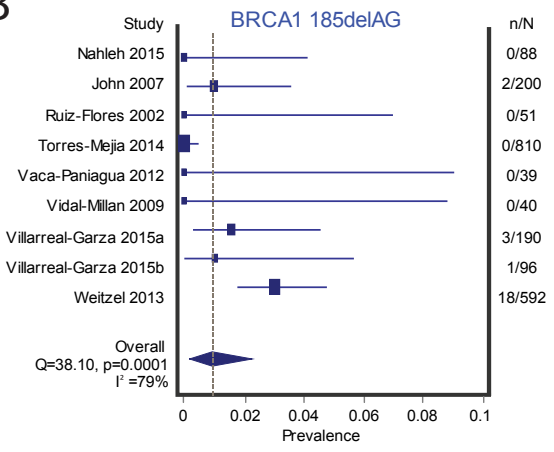

Freq $(95 \% \mathrm{Cl}) \quad \%$ Weight $0.000(0.000-0.041) \quad 10.34$ $0.010(0.001-0.036) \quad 13.06$ $0.000(0.000-0.070) \quad 8.15$ $0.000(0.000-0.005) \quad 15.48$ $0.000(0.000-0.090) \quad 7.07$ $0.000(0.000-0.088) \quad 7.17$ $0.015(0.003-0.045) \quad 12.92$ $0.010(0.000-0.057) \quad 10.67$ $0.030(0.018-0.048) \quad 15.14$

$0.01(0.001-0.022) \quad 100.00$

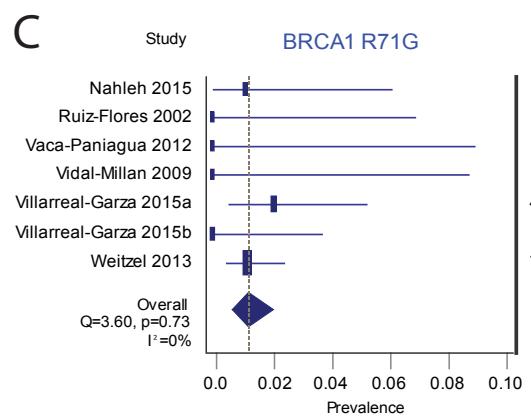

$\mathrm{n} / \mathrm{N} \quad$ Freq $(95 \% \mathrm{Cl}) \quad \%$ Weight

$\begin{array}{lll}1 / 88 & 0.011(0.000-0.061) & 8.12\end{array}$ $0.000(0.000-0.069) \quad 4.72$ $39 \quad 0.000(0.000-0.090) \quad 3.62$ $0 / 40 \quad 0.000(0.000-0.088) \quad 3.71$ $\begin{array}{lll}4 / 190 & 0.021(0.005-0.053) \quad 17.48\end{array}$ $0 / 96 \quad 0.000(0.000-0.037) \quad 8.85$ $7 / 582 \quad 0.012(0.004-0.024) \quad 53.46$ $0.012(0.006-0.019) \quad 100.00$

D

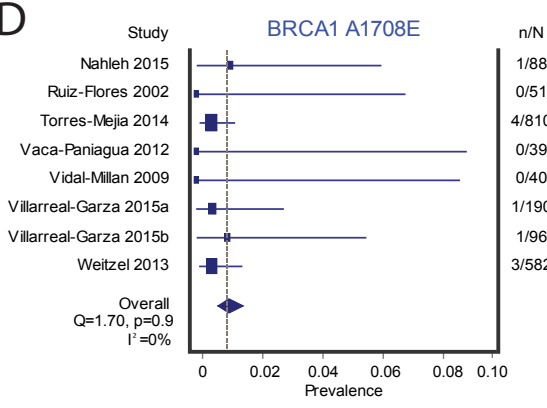

Freq $(95 \% \mathrm{Cl}) \quad \%$ Weight $0.011(0.000-0.061) \quad 4.66$ $0.000(0.001-0.069) \quad 2.71$ $0.004(0.000-0.012) \quad 42.66$ $0.000(0.000-0.090) \quad 2.08$ $0.000(0.000-0.088) \quad 2.13$ $0.005(0.003-0.028) \quad 10.03$ $0.010(0.000-0.056) \quad 5.08$ $0.005(0.018-0.014) \quad 30.66$ $0.010(0.00-0.01) \quad 100.00$

Figure 3: The prevalence for the Mexican subjects for del exon 9.

(0.88\%, 95\% CI: $0.24-1.92 \%$, Figure 4A). The common Ashkenzai Jewish founder mutation 6174delT's prevalence was determined to be $0.32 \%$ (95\% CI: $0.24-0.53 \%$, Figure 4B). Only two mutations had a higher prevalence than 6174delT, E49X (0.38\%, 95\% CI: $0.13-0.75 \%$, Figure $4 \mathrm{C})$ and 3492 ins T (0.32\%, 95\% CI: $0.24-0.53 \%$, Figure $4 \mathrm{D})$. The remaining Forest Plots can be found in supplemental material (Supplemental Figure 3).

Next, we determined if the mutational frequencies were consistent between Mexican and other Latin Americans. The prevalence of the pathogenic BRCA2 mutation among Mexicans was calculated (Table 3). The most prevalent BRCA2 mutations found in Mexican subjects were E49X (0.68\%, 95\% CI: 0.28-1.25\%, Figure 5A), 3492insT (0.60\%, 95\% CI: 0.12-1.36\%, Figure 5B), G273R (0.57\%, 95\% CI: 0.21-1.10\%, Figure 5C), and W2586X (0.56\%, 95\% CI: $0.11-1.33 \%$, Figure 5D). The remaining Forest Plots can be found in supplemental material (Supplemental Figure 4). The 6174delT and $\mathrm{H} 372 \mathrm{H}$ mutations were not found among Mexican studies; however, the 3492insT, G273R, and W2586X mutations were only found in Mexican subjects. The E49X mutation prevalence was not different between Mexicans studies and other Latin American studies ( $\mathrm{p}=0.159)$.

\section{Test for sensitivity and publication bias}

We assessed the publication bias for pathogenic mutation prevalence for BRCA1 and BRCA2 (Figure 6). For BRCA1, the BeggMazumdar's test calculated Kendall's tau to be $0.26(\mathrm{p}=0.036)$, and Egger's test's bias $=2.12$ (95\% CI: 0.69-3.55, $\mathrm{p}=0.005)$. For BRCA2, the Begg-Mazumdar calculated Kendall's tau to be 0.28 ( $\mathrm{p}=0.037)$, and Egger's test's bias $=2.35$ (95\% CI: $1.42-3.28, \mathrm{p}<0.001)$. Examining the funnel plots, two studies (Lara 2012 and Solano 2012) could bias the results. To assess the sensitivity, one study was removed at a time and the effect on the pooled prevalence was reevaluated. For Latin
American subjects, the pooled prevalence was resistance. These results suggest limited bias, which would minimally affect the results of the meta-analysis. The publication bias for each individual mutation was assessed for each meta-analysis and listed in Table 3.

\section{Discussion}

Breast cancer is the most common cancer among females in Latin American countries. While other risk factors, such as high estrogen exposure and age of menarche increases the risk of breast cancer development, mutations in the BRCA1 and BRCA2 genes have a more profound effect in certain population [53]. Genetic testing is an expensive procedure that can aid the development of specific treatment options. Unfortunately, with the large amount of possible mutations to test for, there is a need for a consensus on specific mutations.

In our study, we determined the prevalence of all BRCA1 and BRCA2 mutations among Latin American breast cancer subjects. This study is similar to Wang et al. and Forat-Yazdi et al., who determine the prevalence in breast cancer families and Iranians, respectively [54,55]; however, neither study examined the prevalence in nor contained Latin American subjects, specifically. Furthermore, Wang et al. and ForatYazdi et al. excluded studies from their meta-analysis that determine the absence of a mutation from their sample, which may have led them to overestimated the mutation's prevalence. Here, we did incorporate any report that examined the region for these 80 BRCA1 and 69 BRCA2 pathogenic mutations. For example, Lara 2012 and Solano 2012, the two studies that found BRCA2 H372N, determined the frequency to be $55.2 \%$ and $25.5 \%$, respectively, which would give a pooled prevalence of about $38.8 \%$; however, including reports that determine the absence of this mutation, gives the pooled prevalence of $0.88 \%$. Since many studies failed to determine this mutation in larger samples, this result would suggest that including the negative data would yield a more accurate value. 
A

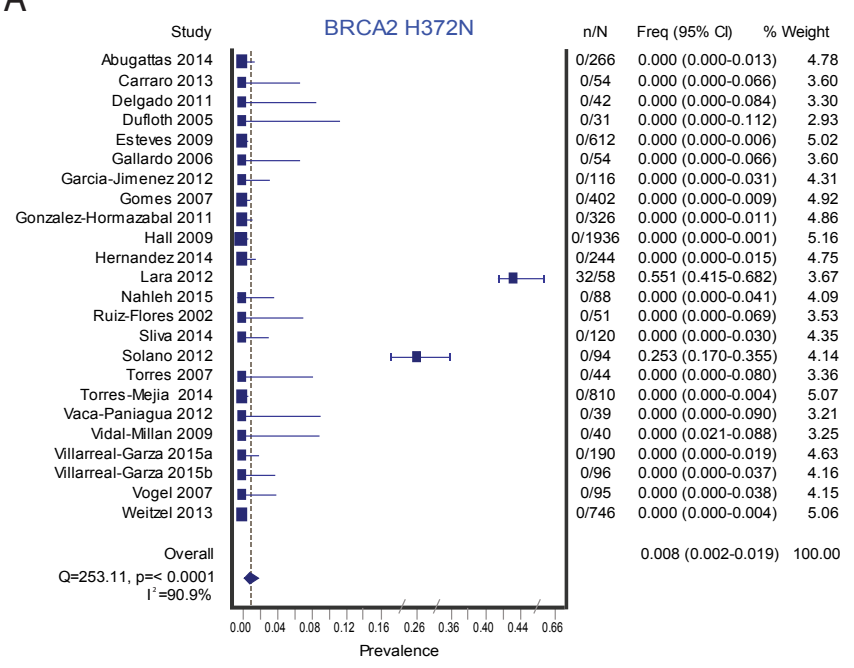

B

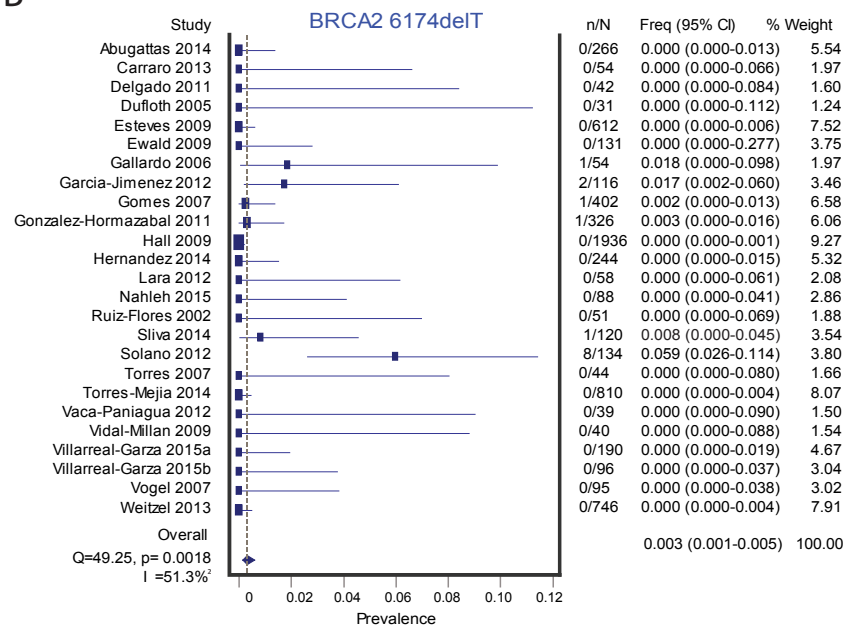

C

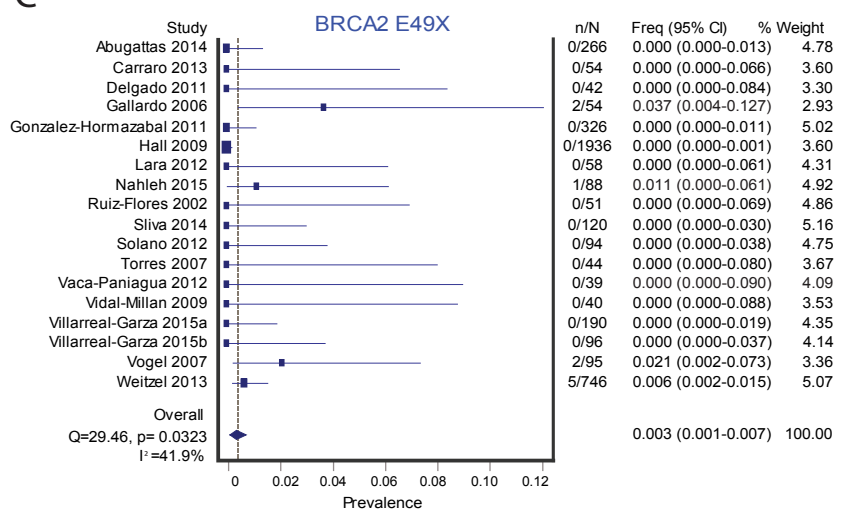

$\mathrm{D}$

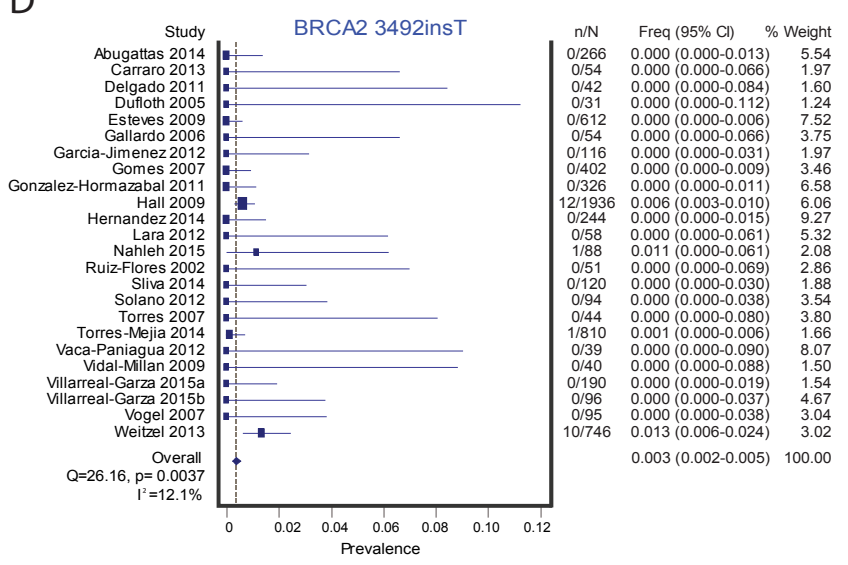

Figure 4: BRCA2 mutations found in Latin American Breast cancer subjects.

The most common BRCA1 mutation for Latin American breast cancer subjects was deletion of exons 9-12. This mutation leads to an inactive form of BRCA1. However, this mutation was only found in Mexican studies and no other country. It is believed to have originated in the state of Puebla [31]; however, Weitzel et al., found this mutation present in subjects that originated from other distant regions of Mexico [21]. This would suggest emigration has led to the dispersion of this mutation. Interestingly, this mutation has not been reported in Southern Mexico or Guatemala, maybe due to a lack of studies. More research is required to determine the regions in Mexico where this mutation is prevalent.

The second most prevalent BRCA1 mutation was 185delAG. Unlike the deletion exon 9-12, the 185delAG mutation has been found in many different regions of Latin America (Argentina, Brazil, Chile, Mexico and Peru). Numerous reports have demonstrated that the BRCA1 185delAG increases the risk of developing breast cancer. This has led to the additional screening of subjects from certain ethnicities for the presence of this mutation, such as the Ashkenazi Jewish descendants. Nonetheless, within the Latin American populations, there is inconsistent evidence about the prevalence of this mutation in breast cancer subjects, which ranged from $0.0 \%$ (19 studies) to $5.22 \%$. Here, we provide evidence that 185 delAG frequency was significantly prevalent and was not different between Mexico and all other Latin American countries $(0.90 \%$ vs. $0.94 \%, p=0.70)$; then again, not all countries in Central and South America were properly represented. A similar result was determined for BRCA1 A1708E and BRCA2 E49X. On the other hand, BRCA1 R71G was found in Mexicans as well as Argentinians but was more prevalent in Mexico. Moreover, BRCA2 6174delT and H372N were found in Argentina, Brazil, Chile, Costa Rica, and Venezuela and not Mexico. As well, the BRCA2 3492insT, G273R, and W2586X were found only in Mexico and not any other Latin American country. These examples beg the question that should there be a select set of mutations examined for certain regions of Latin America. Furthermore, it demonstrates that specific regions of Latin American are associated with certain BRCAs mutations.

Central and South America were settled by many different groups from the Iberian Peninsula. We posit that due to emigration from certain regions of Europe has led to the presence of certain mutations in specific regions of Latin America. For example, the BRCA2 3492insT 

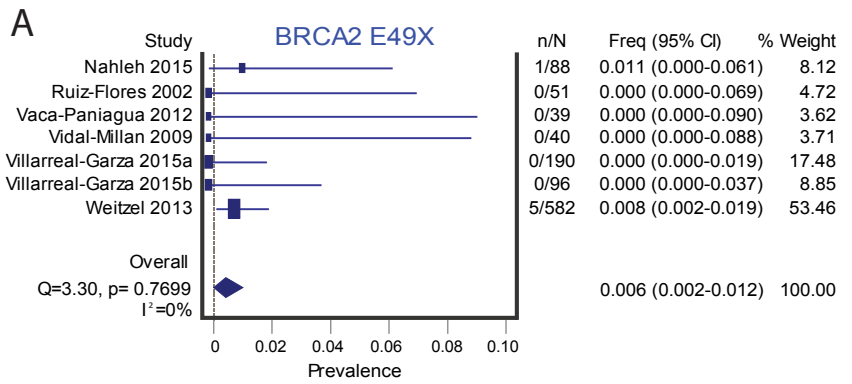

B

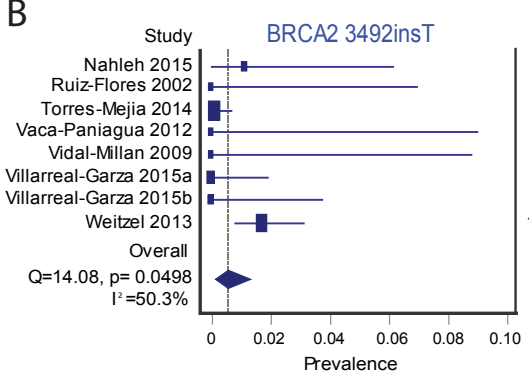

C

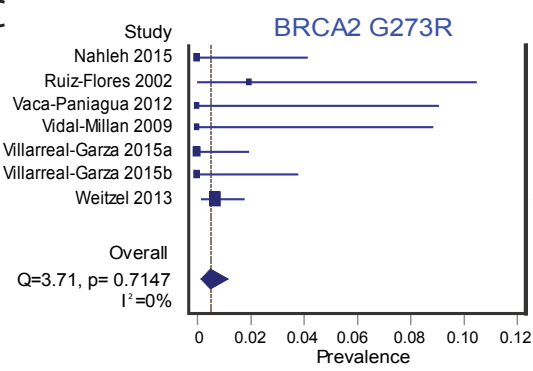

$\mathrm{n} / \mathrm{N} \quad$ Freq $(95 \% \mathrm{Cl}) \quad \%$ Weight

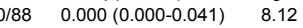
\begin{tabular}{lll}
$1 / 51$ & $0.019(0.000-0.104)$ & 4.72 \\
\hline & 0.300
\end{tabular} $\begin{array}{lll}0 / 39 & 0.000(0.000-0.090) & 3.62 \\ 0.40 & 0.000(0.000-0.088) & 3.71\end{array}$

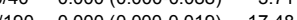
(0/96 $0.000(0.000-0.019) \quad 17.48$ $\begin{array}{ll}1582 & 0.006(0.001-0.017) \quad 53.46\end{array}$

$0.005(0.002-0.011) \quad 100.00$

Figure 5: Prevalent BRCA2 mutations found in Mexican subjects.

A
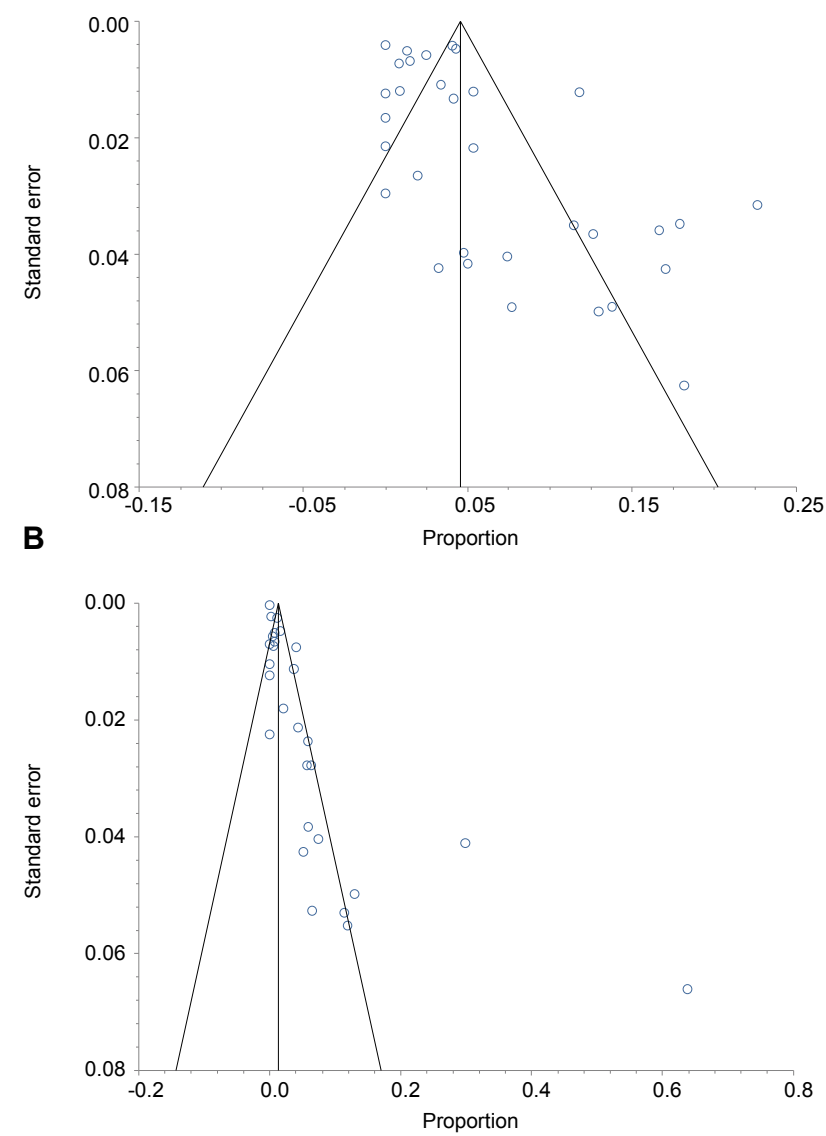

Figure 6: Pathogenic mutation prevalence for BRCA1 and BRCA2. polymorphism has been identified in many regions of Spain: Austurias [56], Valencia [57-60], Aragon [60], Castilla-Leon [61], and Madrid [62], where the mutation frequency ranged between $0.22-2.08 \%$. Interestingly, this mutation has not been reported in Barcelona or Galicia [63], suggesting that this mutation originated from a specific region of Spain. In Mexico, a majority of the population ancestry is from Spain, implying that the BRCA2 3492insT could be found in Mexico. Indeed, this meta-analysis does provide evidence that BRCA2 3492insT was significantly present among Mexicans. A majority of the reports focused on Mexican subjects used in this meta-analysis failed to determine the presence of BRCA2 3492insT. These reports focused on highly-populated regions of Mexico City, the State of Mexico, Nuevo Leon and Veracruz and surrounding regions-most of the states located in the center of Mexico. However, Weitzel et al. did observe the mutation in subjects from Durango, Guerrero, Jalisco, Sinaloa, Sonora, and Zacatecas [21]. With the exception of Guerrero, these states are located in Western Mexico, which would posit the notion that these states present this mutation due to a more pronounced Spanish influence. This is supported by the work of Moreno-Estrada et al., which indicates that subjects from Western Mexico do have a greater Spanish genetic composition than states from Eastern or Southern Mexico [64]. Interestingly, the BRCA2 3492ins T mutation has not been observed in other Central or South American countries [15,17,33,39,43,47-50]. As seen with the San Luis Valley studies, due to Spaniard expeditions, the BRCA1 185delAG mutation was introduced and spread among the region, leading to its extraordinary prevalence $[65,66]$. This would also support the notion that the BRCA2 3492insT mutation is specific for Mexico and Spain. Overall, the region-specific mutational patterns are likely caused by original differences of the native population and induced differences by emigration.

In this meta-analysis there are a few limitations. First, the included studies collected genomic DNA from either blood or a buccal sample, or both. Recent reports have suggest that a blood sample is the superior method, suggesting that studies that used a buccal sample could 
Citation: Porchia LM, Gonzalez-Mejia ME, Calderilla-Barbosa L, Ordaz-Diaz N, Islas F, et al. (2015) Common BRCA1 and BRCA2 Mutations among Latin American Breast Cancer Subjects: A Meta-Analysis. J Carcinogene Mutagene 6: 228. doi:10.4172/2157-2518.1000228

underestimate or fail to determine the prevalence of BRCA mutations. Second, studies with smaller sample sizes does increase the prevalence of a single case found but also decrease the chance of discovering a positive case among the sample. Third, due to the emigration pattern that has led to the high diversity of Latin America, many regions of Central and South America are under-represented. For example, as mentioned above, the 3492ins T mutation was mainly found on the Western part of Mexico and not in the Northern or the Eastern regions or any other Latin American country. This result demonstrates the need for more region-specific analyses.

In conclusion, this study identifies the most prevalent BRCA1 and BRCA2 mutations found in Latin American breast cancer subjects. Furthermore, we demonstrate that certain mutations are only specific for certain regions, whereas others are constant throughout Latin America. This information will aid in developing a more narrow genetic screening strategy based on the subject's background and lead to cheaper testing. However, with most Latin American countries have not been assessed for BRCA1 and BRCA2 mutations, further studies are required.

\section{Acknowledgment}

We would like to express our gratitude to Ricardo Villegas-Tovar, from BUAP Libraries-Department, for aiding in literature searches and finding article, and Alfredo Mendez for reviewing this manuscript.

\section{References}

1. Molina Y, Thompson B, Espinoza N, Ceballos R (2013) Breast cancer interventions serving US-based Latinas: current approaches and directions. Women's health 9: 335-48.

2. Hortobagyi GN, de la Garza Salazar J, Pritchard K, Amadori D, Haidinger R, et al. (2005) The global breast cancer burden: variations in epidemiology and survival. Clin Breast Cancer 6: 391-401.

3. Justo N, Wilking N, Jönsson B, Luciani S, Cazap E (2013) A review of breast cancer care and outcomes in Latin America. Oncologist 18: 248-256.

4. Li Cl, Malone KE, Daling JR (2003) Differences in breast cancer stage, treatment, and survival by race and ethnicity. Arch Intern Med 163: 49-56.

5. Ferlay J, Shin HR, Bray F, Forman D, Mathers C, et al. (2010) Estimates of worldwide burden of cancer in 2008: GLOBOCAN 2008. Int J Cancer 127 2893-2917.

6. Coughlin SS, Ekwueme DU (2009) Breast cancer as a global health concern. Cancer Epidemiol 33: 315-318.

7. Anderson BO, Jakesz R (2008) Breast cancer issues in developing countries: an overview of the Breast Health Global Initiative. World J Surg 32: 2578-2585.

8. Ford D, Easton DF, Stratton M, Narod S, Goldgar D, et al. (1998) Genetic heterogeneity and penetrance analysis of the BRCA1 and BRCA2 genes in breast cancer families. The Breast Cancer Linkage Consortium. American journal of human genetics 62 : $676-89$.

9. Weitzel JN, Blazer KR, MacDonald DJ, Culver JO, Offit K (2011) Genetics, genomics, and cancer risk assessment: State of the Art and Future Directions in the Era of Personalized Medicine. CA Cancer J Clin 61: 327-359.

10. Antoniou A, Pharoah PD, Narod S, Risch HA, Eyfjord JE, et al. (2003) Average risks of breast and ovarian cancer associated with BRCA1 or BRCA2 mutations detected in case Series unselected for family history: a combined analysis of 22 studies. Am J Hum Genet 72: 1117-1130.

11. Narod SA (2009) Screening for BRCA1 and BRCA2 mutations in breast cancer patients from Mexico: the public health perspective. Salud Publica Mex 51 Suppl 2: s191-196.

12. de Juan I, Esteban E, Palanca S, Barragán E, Bolufer $P$ (2009) High-resolution melting analysis for rapid screening of BRCA1 and BRCA2 Spanish mutations. Breast Cancer Res Treat 115: 405-414.

13. Díez Gibert O (2006) ESTUDIO MOLECULAR DE LOS GENES BRCA1 Y BRCA2 EN CÁNCER DE MAMA HEREDITARIO. Ed Cont Lab Clín 9: 19-27.
14. Díez Gibert O, Cornet Ciurana M, Gutiérrez Enríquez $S$, Domènech Maria $M$ RyCA T, et al. (2007) Estudio de los genes BRCA1 y BRCA2 en 200 familias con cáncer de mama hereditario. Química Clínica 26: 202-206.

15. Dutil J, Colon-Colon JL, Matta JL, Sutphen R, Echenique M (2012) Identification of the prevalent BRCA1 and BRCA2 mutations in the female population of Puerto Rico. Cancer Genet 205: 242-248.

16. Hall MJ, Reid JE, Burbidge LA, Pruss D, Deffenbaugh AM, et al. (2009) BRCA1 and BRCA2 mutations in women of different ethnicities undergoing testing for hereditary breast-ovarian cancer. Cancer 115: 2222-2233.

17. Lara K, Consigliere N, Pérez J, Porco A (2012) BRCA1 and BRCA2 mutations in breast cancer patients from Venezuela. Biol Res 45: 117-130.

18. Ruiz-Flores P, Sinilnikova OM, Badzioch M, Calderon-Garcidueñas AL, Chopin $\mathrm{S}$, et al. (2002) BRCA1 and BRCA2 mutation analysis of early-onset and familial breast cancer cases in Mexico. Hum Mutat 20: 474-475.

19. Vaca-Paniagua F, Alvarez-Gomez RM, Fragoso-Ontiveros V, Vidal-Millan S, Herrera LA, et al. (2012) Full-exon pyrosequencing screening of BRCA germline mutations in Mexican women with inherited breast and ovarian cancer. PloS one 7: e37432.

20. Vogel KJ, Atchley DP, Erlichman J, Broglio KR, Ready KJ, et al. (2007) BRCA1 and BRCA2 genetic testing in Hispanic patients: mutation prevalence and evaluation of the BRCAPRO risk assessment model. J Clin Oncol 25: 46354641.

21. Weitzel JN, Clague J, Martir-Negron A, Ogaz R, Herzog J, et al. (2013) Prevalence and type of BRCA mutations in Hispanics undergoing genetic cancer risk assessment in the southwestern United States: a report from the Clinical Cancer Genetics Community Research Network. J Clin Oncol 31: 210 216

22. Weitzel JN, Lagos V, Blazer KR, Nelson R, Ricker C, et al. (2005) Prevalence of BRCA mutations and founder effect in high-risk Hispanic families. Cancer Epidemiol Biomarkers Prev 14: 1666-1671.

23. Kendall MG, Stuart A, Ord K, Arnold S, O'Hagan A (1994) Kendall's Advanced Theory of Statistics, Classical Inference and the Linear Model. Wiley.

24. Miller JJ (1978) The inverse of the Freeman-Tukey double arcsine transformation. American Statistician 32: 138-138.

25. DerSimonian R, Laird N (1986) Meta-analysis in clinical trials. Control Clin Trials 7: 177-188.

26. Begg CB, Mazumdar M (1994) Operating characteristics of a rank correlation test for publication bias. Biometrics 50: 1088-1101.

27. Egger M, Davey Smith G, Schneider M, Minder C (1997) Bias in meta-analysis detected by a simple, graphical test. BMJ 315: 629-634.

28. Nahleh Z, Otoukesh S, Dwivedi AK, Mallawaarachchi I, Sanchez L, et al. (2014) Clinical and pathological characteristics of Hispanic BRCA-associated breast cancers in the American-Mexican border city of El Paso, TX. Am J Cancer Res 5: $466-471$.

29. Torres-Mejia G, Royer R, Llacuachaqui M, Akbari MR, Giuliano AR, et al (2015) Recurrent BRCA1 and BRCA2 mutations in Mexican women with breas cancer. Cancer Epidemiol Biomarkers Prev 24: 498-505.

30. Vidal-Millán S, Taja-Chayeb L, Gutiérrez-Hernández O, Ramírez Ugalde MT, Robles-Vidal C, et al. (2009) Mutational analysis of BRCA1 and BRCA2 genes in Mexican breast cancer patients. Eur J Gynaecol Oncol 30: 527-530.

31. Villarreal-Garza C, Alvarez-Gómez RM, Pérez-Plasencia C, Herrera LA Herzog J, et al. (2015) Significant clinical impact of recurrent BRCA1 and BRCA2 mutations in Mexico. Cancer 121: 372-378.

32. Villarreal-Garza C, Weitzel JN, Llacuachaqui M, Sifuentes E, MagallanesHoyos MC, et al. (2015) The prevalence of BRCA1 and BRCA2 mutations among young Mexican women with triple-negative breast cancer. Breast Cancer Res Treat 150: 389-394.

33. Carraro DM, Koike Folgueira MA, Garcia Lisboa BC, Ribeiro Olivieri EH Vitorino Krepischi AC, et al. (2013) Comprehensive analysis of BRCA1, BRCA2 and TP53 germline mutation and tumor characterization: a portrait of earlyonset breast cancer in Brazil. PloS one 8: e57581.

34. Dufloth RM, Carvalho S, Heinrich JK, Shinzato JY, dos Santos CC, et al. (2005) Analysis of BRCA1 and BRCA2 mutations in Brazilian breast cancer patients with positive family history. Revista paulista de medicina 123: 192-197. 
Citation: Porchia LM, Gonzalez-Mejia ME, Calderilla-Barbosa L, Ordaz-Diaz N, Islas F, et al. (2015) Common BRCA1 and BRCA2 Mutations among Latin American Breast Cancer Subjects: A Meta-Analysis. J Carcinogene Mutagene 6: 228. doi:10.4172/2157-2518.1000228

35. Esteves VF, Thuler LC, Amendola LC, Koifman RJ, Koifman S, et al. (2009) Prevalence of BRCA1 and BRCA2 gene mutations in families with medium and high risk of breast and ovarian cancer in Brazil. Braz J Med Biol Res 42: 453-457.

36. Ewald IP, Izetti P, Vargas FR, Moreira MA, Moreira AS, et al. (2011) Prevalence of the BRCA1 founder mutation c.5266dupin Brazilian individuals at-risk for the hereditary breast and ovarian cancer syndrome. Hered Cancer Clin Pract 9: 12.

37. Gomes MC, Costa MM, Borojevic R, Monteiro AN, Vieira R, et al. (2007) Prevalence of BRCA1 and BRCA2 mutations in breast cancer patients from Brazil. Breast Cancer Res Treat 103: 349-353.

38. Silva FC, Lisboa BC, Figueiredo MC, Torrezan GT, Santos EM, et al. (2014) Hereditary breast and ovarian cancer: assessment of point mutations and copy number variations in Brazilian patients. BMC medical genetics 15: 55.

39. Gallardo M, Silva A, Rubio L, Alvarez C, Torrealba C, et al. (2006) Incidence of BRCA1 and BRCA2 mutations in 54 Chilean families with breast/ovarian cancer, genotype-phenotype correlations. Breast cancer research and treatment 95: 81-87.

40. Gonzalez-Hormazabal P, Gutierrez-Enriquez S, Gaete D, Reyes JM, Peralta O, et al. (2011) Spectrum of BRCA1/2 point mutations and genomic rearrangements in high-risk breast/ovarian cancer Chilean families. Breast cancer research and treatment 126: 705-716.

41. Sanchez A, Faundez P, Carvallo $P$ (2011) Genomic rearrangements of the BRCA1 gene in Chilean breast cancer families: an MLPA analysis. Breast Cancer Res Treat 128: 845-853.

42. Trincado P, Fardella C, Mayerson D, Montero L, O'Brien A, et al. (1999) [Prevalence of the $185 \mathrm{Ag}$ deletion of the BRCA1 gene in Chilean women with breast neoplasm]. Rev Med Chil 127: 19-22.

43. Hernández JE, Llacuachaqui M2, Palacio GV1, Figueroa JD3, Madrid J4, et al. (2014) Prevalence of BRCA1 and BRCA2 mutations in unselected breast cancer patients from medellín, Colombia. Hered Cancer Clin Pract 12: 11.

44. Sanabria MC, Munioz G, Vargas $\mathrm{Cl}$ (2009) Mutations in the BRCA1 gene (185delAG and 5382insC) are not present in any of the 30 breast cancer patients analyzed from eastern Colombia. Biomedica 29: 61-72.

45. Torres D, Rashid MU, Gil F, Umana A, Ramelli G, et al. (2007) High proportion of BRCA1/2 founder mutations in Hispanic breast/ovarian cancer families from Colombia. Breast Cancer Res Treat 103: 225-232.

46. Torres D, Rashid MU; Colombian Breast Cancer Study Group (COLBCS), Seidel-Renkert A, Weitzel JN, Briceno I, et al. (2009) Absence of the BRCA1 del (exons 9-12) mutation in breast/ovarian cancer families outside of Mexican Hispanics. Breast Cancer Res Treat 117: 679-681.

47. Solano AR, Aceto GM, Delettieres D, Veschi S, Neuman MI, et al. (2012) BRCA1 And BRCA2 analysis of Argentinean breast/ovarian cancer patients selected for age and family history highlights a role for novel mutations of putative south-American origin. SpringerPlus 1: 20 .

48. Garcia-Jimenez L, Gutierrez-Espeleta G, Narod SA (2012) Descriptive epidemiology and molecular genetics of hereditary breast cancer in Costa Rica. Revista de biologia tropical 60: 1663-1668.

49. Abugattas $J$, Llacuachaqui $M$, Allende $Y S$, Velásquez $A A$, Velarde $R$, et al (2014) Prevalence of BRCA1 and BRCA2 mutations in unselected breast cancer patients from Peru. Clin Genet .

50. Delgado L, Fernández G, Grotiuz G, Cataldi S, González A, et al. (2011) BRCA1 and BRCA2 germline mutations in Uruguayan breast and breastovarian cancer families. Identification of novel mutations and unclassified variants. Breast Cancer Res Treat 128: 211-218.

51. Anton-Culver H, Cohen PF, Gildea ME, Ziogas A (2000) Characteristics of BRCA1 mutations in a population-based case series of breast and ovarian cancer. Eur J Cancer 36: 1200-1208.

52. John EM, Miron A, Gong G, Phipps Al, Felberg A, et al. (2007) Prevalence of pathogenic BRCA1 mutation carriers in 5 US racial/ethnic groups. JAMA 298: 2869-2876.
53. Haimov-Kochman R, Lavy $Y$, Hochner-Celinkier D (2002) Review of risk factors for breast cancer--what's new?. Harefuah 141: 702-708, 761.

54. Forat-Yazdi $M$, Neamatzadeh $H$, Sheikhha $M H$, Zare-Shehneh $M$, Fattahi M (2015) BRCA1 and BRCA2 common mutations in iranian breast cancer patients: a meta analysis. Asian Pac J Cancer Prev 16: 1219-1224.

55. Wang F, Fang Q, Ge Z, Yu N, Xu S, et al. (2012) Common BRCA1 and BRCA2 mutations in breast cancer families: a meta-analysis from systematic review. Mol Biol Rep 39: 2109-2118.

56. Blay P, Santamaría I, Pitiot AS, Luque M, Alvarado MG, et al. (2013) Mutationa analysis of BRCA1 and BRCA2 in hereditary breast and ovarian cancer families from Asturias (Northern Spain). BMC Cancer 13: 243.

57. Bolufer P, Munárriz B, Santaballa A, Velasco E, Lerma E, et al. (2005) [BRCA1 and BRCA2 mutations in patients with familial breast cancer]. Med Clin (Barc) 124: $10-12$.

58. de Juan Jimenez I, Garcia Casado Z, Palanca Suela S, Esteban Cardenosa E, Lopez Guerrero JA, et al. (2013) Novel and recurrent BRCA1/BRCA2 mutations in early onset and familial breast and ovarian cancer detected in the Program of Genetic Counseling in Cancer of Valencian Community (eastern Spain). Fam Cancer 12: 767-777.

59. Esteban Cardenosa E, Bolufer Gilabert P, de Juan Jimenez I, Palanca Suela S, Barragan Gonzalez E, et al. (2010) Broad BRCA1 and BRCA2 mutational spectrum and high incidence of recurrent and novel mutations in the eastern Spain population. Breast Cancer Res Treat 121: 257-260.

60. Miramar MD, Calvo MT, Rodriguez A, Antón A, Lorente F, et al. (2008) Genetic analysis of BRCA1 and BRCA2 in breast/ovarian cancer families from Aragon (Spain): two novel truncating mutations and a large genomic deletion in BRCA1. Breast Cancer Res Treat 112: 353-358.

61. Infante M, Duran M, Esteban-Cardenosa E, Miner C, Velasco E (2006) High proportion of novel mutations of BRCA1 and BRCA2 in breast/ovarian cancer patients from Castilla-Leon (central Spain). J Hum Genet 51: 611-617.

62. de la Hoya M, Osorio A, Godino J, Sulleiro S, Tosar A, et al. (2002) Association between BRCA1 and BRCA2 mutations and cancer phenotype in Spanish breast/ovarian cancer families: implications for genetic testing. Int $\mathrm{J}$ Cancer 97: 466-471.

63. xDiez O, Gutierrez-Enriquez S, Balmana J (2010) Heterogeneous prevalence of recurrent BRCA1 and BRCA2 mutations in Spain according to the geographical area: implications for genetic testing. Fam Cancer 9: 187-191.

64. Moreno-Estrada A, Gignoux CR, Fernandez-Lopez JC, Zakharia F, Sikora M et al. (2014) Human genetics. The genetics of Mexico recapitulates Native American substructure and affects biomedical traits. Science 344: 1280-1285.

65. Makriyianni I, Hamel N, Ward S, Foulkes WD, Graw S (2005) BRCA1:185delAG found in the San Luis Valley probably originated in a Jewish founder. J Med Genet 42: e27.

66. Mullineaux LG, Castellano TM, Shaw J, Axell L, Wood ME, et al. (2003) Identification of germline 185delAG BRCA1 mutations in non-Jewish Americans of Spanish ancestry from the San Luis Valley, Colorado. Cancer 98: 597-602. 